\title{
The Influence of Mechanical Constraints Introduced by $\beta$ Annealed Microstructures on the Yield Strength and Ductility of Ti-6Al-4V
}

\author{
Matthew Kasemer ${ }^{1,2}$, Romain Quey ${ }^{3}$, and Paul Dawson ${ }^{1}$ \\ ${ }^{1}$ Sibley School of Mechanical and Aerospace Engineering, Cornell University, Ithaca, NY, USA \\ ${ }^{2}$ Cornell High Energy Synchrotron Source, Cornell University, Ithaca, NY, USA \\ ${ }^{3}$ École des Mines de Saint-Étienne, CNRS UMR 5307, Saint-Étienne, France
}

\begin{abstract}
Discussed is a computational study of the influence of the microstructure's geometric morphology on the yield strength and ductility of Ti-6Al-4V. Uniaxial tension tests were conducted on physical specimens to determine the macroscopic yield strength and ductility of two microstructural variations (mill annealed and $\beta$ annealed) to establish comparisons of macroscopic properties. A multi-experimental approach was utilized to gather two dimensional and three dimensional data, which were used to inform the construction of representative $\beta$ annealed polycrystals. A highly parallelized crystal plasticity finite element framework was employed to model the deformation response of the generated polycrystals subjected to uniaxial tension. To gauge the macroscopic response's sensitivity to the morphology of the geometry, the key geometrical features - namely the number of high temperature $\beta$ phase grains, $\alpha$ phase colonies, and size of remnant secondary $\beta$ phase lamellae - were altered systematically in a suite of simulations. Both single phase and dual phase aggregates were studied. Presented are the calculated yield strengths and ductilities, and the resulting trends as functions of geometric parameters are examined in light of the heterogeneity in deformation at the crystal scale.
\end{abstract}

\section{Introduction}

Ti-6Al-4V is the most widely used titanium alloy, and is commonly used for critical components in many different engineering applications - desirable because of the concurrence of high strength, corrosion resistance, and low weight [1]. The thermomechanical processes the raw material is subjected to during manufacture have the potential to introduce a wide variety of microstructures, in turn altering the deformation response of the material. Representing two extremes of attainable microstructures are the mill annealed microstructure and the $\beta$ annealed microstructure. Experimental observation has shown that the two microstructures are markedly different in terms of their geometric features and deformation response $[2,3]$. The presence of two crystallographic phases, including a hexagonal crystallographic phase that exhibits strong elastic and plastic anisotropy $[4,5]$ adds complexity to the material's deformation response. How the microstructure effects the deformation response can be understood by considering the mechanical constraints that exist between the two crystallographic phases when equilibrium and compatibility are enforced. Understanding the microstructure-property relationship - specifically in regards to the estimation of the yield strength and ductility - is crucial in an alloy with such ubiquity, wide microstructural variability, and complex crystal scale behavior.

(C) 2016. This manuscript version is made available under the Elsevier user license

http://www.elsevier.com/open-access/userlicense/1.0/ 
Simulating the deformation of these microstructures provides insight into the development of plasticity at the crystal scale, and the influence that the microstructure has on the resulting macroscopic behavior. Mathematical frameworks exist that describe the behavior of plastically deforming materials at the crystal scale $[6,7]$. These crystal plasticity equations are often implemented in a finite element computational framework [8]. Due to constraints on computational capabilities, studies have historically been limited to simplified representations of microstructures [9-11], idealized models for the study of texture evolution [12], or detailed microstructural representations of limited scale [13]. Indeed, while other modeling techniques (e.g. mean field approaches such as the Taylor model or Sachs model) are able to estimate an aggregate's macroscopic response, high fidelity finite element modeling allows for a better understanding of the microstructure's influence on such a response by considering the spatial arrangement of grains and phases. The development of more efficient computational architectures, coupled with the use of highly parallelized code has allowed for the use of larger meshes, and thus the inclusion of fine geometric detail. Recent experimental and computational studies suggest that deformation occurs heterogeneously across individual grains, necessitating the use of high fidelity representations of microstructures in order to accurately predict the deformation response $[14,15]$.

Representation of a microstructure for use in such simulations is dependent on the experimental identification of key geometric features. Two dimensional methods, such as optical microscopy and EBSD provide detailed depictions of surface features of microstructures, yet are lacking due to their failure to elucidate three dimensional features. Recent developments in both destructive [16] and non-destructive [17] orientation mapping techniques allow for the construction of three dimensional maps - providing not only detailed information about the geometric features of a microstructure, but also the spatial distribution of crystallographic orientations. A multi-experimental approach allows for a better understanding of a microstructure's geometric morphology. These data, in turn, may be used to inform the generation of a potentially infinite number of randomly generated microstructure representations that contain features similar to those observed. Voronoi tessellations have proven to be an effective way to represent generic polycrystals $[18,19]$, and techniques and implementation have recently evolved to efficiently facilitate the creation of large polycrystals and attendant robust finite element meshes [20]. While sufficient in representing main features of the mill annealed microstructure, Voronoi tessellations alone are insufficient in accurately representing the fine details of the $\beta$ annealed microstructure.

In this paper, we focus specifically on how attributes of the $\beta$ annealed microstructure introduce mechanical constraints that affect the material's macroscopic properties. A method is described that creates an idealized version of the $\beta$ annealed microstructure that includes the experimentally observed fine geometric features. A highly parallelized crystal plasticity finite element framework is used to simulate the deformation response. Details of the geometry are altered to gauge their influence on macroscopic behavior, and lower bound estimates on the yield strength and ductility are found. Following presentation of the macroscopic properties, concurrent responses at the crystal scale are examined. The influence of structure-imposed constraints within the microstructure on the intensity of deformation heterogeneity is demonstrated with contrasting images of evolving deformation rate banding for defining features of the microstructure. Further, the propensity for microstructure features to constrain slip activity to one or two systems is investigated in detail. The differences in responses at the crystal scale provide a better understanding of the particular features that most influence the trends observed at the macroscopic scale. 


\section{Background}

\subsection{Experimental Observations}

The two crystallographic phases present in Ti-6Al-4V are the $\alpha$ phase, which exhibits hexagonal close packed crystal symmetry, and a secondary $\beta$ phase, which exhibits body centered cubic crystal symmetry. These two phases represent approximately $92 \%$ and $8 \%$ of the volume, respectively, in both the mill annealed and the $\beta$ annealed microstructures. The mill annealed microstructure is characterized by relatively small $(\mathrm{O}(10 \mu \mathrm{m}))$ equiaxed $\alpha$ phase grains with laths of $\beta$ phase between grains. The $\beta$ annealed microstructure is a complex network of thin $\beta$ phase lamellae embedded in $\alpha$ phase transformation structures - or colonies - that form within large $(\mathrm{O}(500 \mu \mathrm{m}))$ equiaxed $\beta$ phase grains present above the transis temperature. Figure 1 displays optical micrographs of both microstructures.

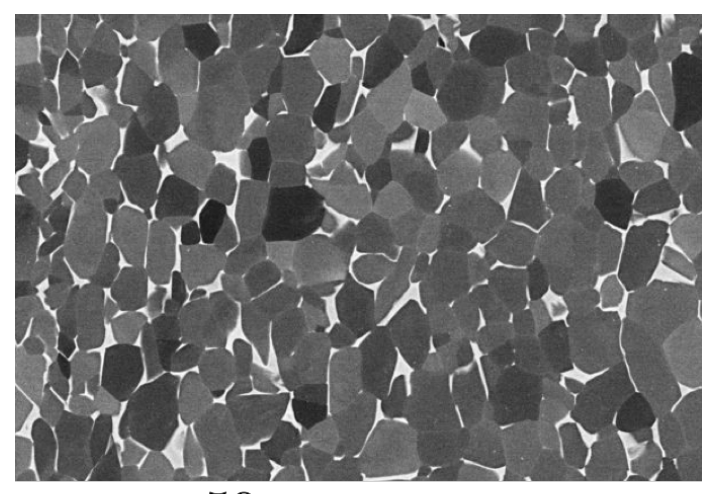

$50 \mu m$

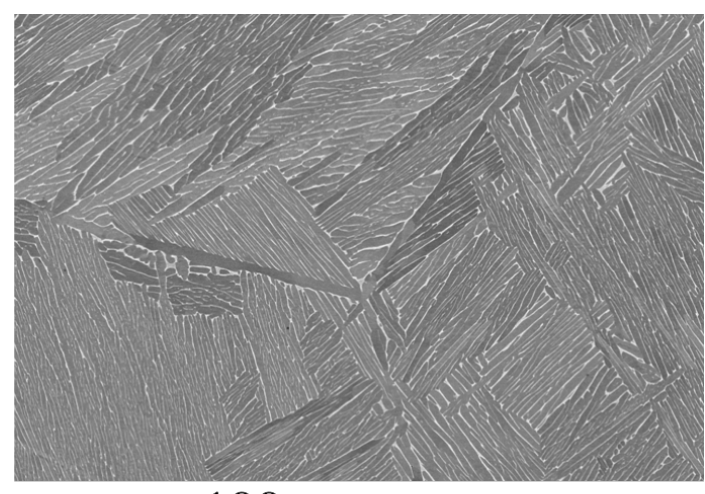

$100 \mu m$

(a)

(b)

Figure 1: Optical micrographs of the (a) mill annealed microstructure, and (b) $\beta$ annealed microstructure. Darker regions of the micrographs are regions of $\alpha$ phase, and lighter regions represent $\beta$ phase. (Reproduced from [1].)

\subsubsection{Macroscopic Tensile Tests}

Tensile tests were performed on samples with a $6.35 \mathrm{~mm}$ diameter circular cross section and gage length of $31.75 \mathrm{~mm}$ in order to characterize the differences in the yield strength and ductility between the two microstructures. Eight mill annealed samples were fabricated from a rolled sheet, each with its tensile direction aligned with the rolling direction, and eight $\beta$ annealed samples were fabricated from a separate rolled sheet, each with its tensile direction aligned with the rolling direction. Tensile tests were conducted monotonically at an engineering strain rate of $0.001 \mathrm{~s}^{-1}$. Yield strength was calculated from the engineering stress-strain curve using a $0.1 \%$ offset.

A practical measure of ductility was chosen as the engineering strain at which the sample incurred the maximum engineering stress, or maximum load, which forms the basis for the Considère condition for stable deformation. Additionally, the amount of hardening - quantified as the difference between the ultimate tensile strength (the stress at ductility) and the yield strength - is included. Spreads in the offset yield strength, ductility, and hardening are calculated as each set's standard deviation. Table 1 details the aggregate results of these tensile tests, while Figure 2 shows example stress-strain curves for both microstructures. The $\beta$ annealed microstructure exhibits a lower yield strength, a significantly lower ductility, and a larger amount of hardening than the mill 
annealed microstructure, as well as a larger degree of variability in all measures. Qualitatively, the mill annealed samples were smooth at the onset of necking, while the $\beta$ annealed samples exhibited pronounced surface roughness (i.e., "orange-peel") - indicating the presence of large grains.

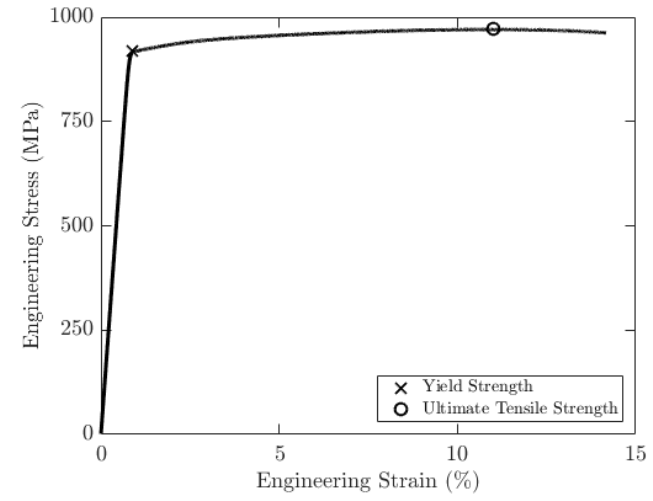

(a)

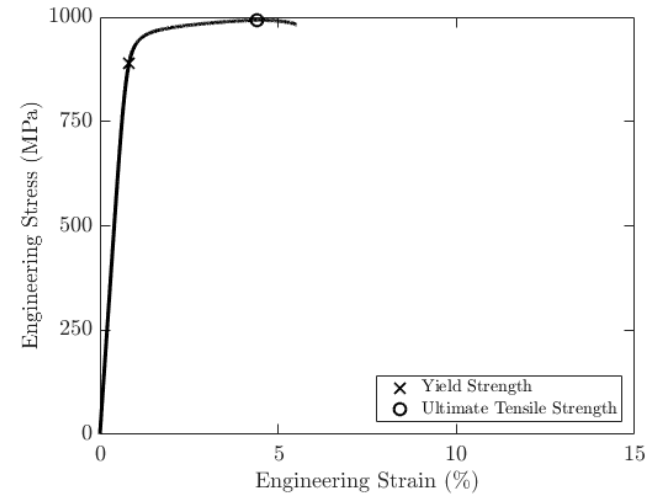

(b)

Figure 2: Example experimental stress-strain curves for the (a) mill annealed microstructure and the (b) $\beta$ annealed microstructure.

\begin{tabular}{cccc}
\hline Microstructure & Yield Strength (MPa) & Ductility (\%) & Hardening (MPa) \\
\hline Mill Annealed & $909.0 \pm 4.8$ & $11.6 \pm 0.7$ & $64.0 \pm 18.3$ \\
$\beta$ Annealed & $898.6 \pm 30.5$ & $4.3 \pm 1.1$ & $96.6 \pm 22.9$ \\
\hline
\end{tabular}

Table 1: Yield strength, ductility, and hardening calculated from macroscopic tensile tests.

\subsubsection{High Energy Diffraction Microscopy}

To gain insight on the three dimensional geometric morphology of the $\beta$ annealed microstructure, a high energy X-ray diffraction experiment was performed by other investigators working on this same material [21]. A sample with a $1 \mathrm{~mm}$ square cross section was interrogated. This sample was cut from the same rolled sheet as the macroscopic tensile test samples. Diffraction peaks were recorded in the near field regime - that is, with the detector sufficiently close to the sample such that both crystallographic orientation and spatial location associated with a Bragg peak may be deduced [17]. An X-ray beam of width $1.2 \mathrm{~mm}$ and height $2 \mu \mathrm{m}$ was used to illuminate a planar slice of the sample, and a spatial map of orientations for the plane was deduced. The sample was successively translated through the beam such that a total of 30 planar orientation maps were available. When stacked, these planar maps form a three dimensional spatial map of orientations (with a total height of $0.75 \mathrm{~mm}$ ). Detector resolution was such that only $\alpha$ phase structures were characterized experimentally (Figure 3(a)).

Because the $\alpha$ phase colonies are transformation structures within grains comprised solely of $\beta$ phase crystals present when the material was rolled above the transis temperature, the morphology of the prior $\beta$ phase (Figure 3(b)) could be determined based on established methods [22]. Qualitatively, the pre-transformation $\beta$ phase structure was shown to have relatively large equiaxed grains with angles of $\sim 120^{\circ}$ at triple junctions. This suggests an equilibrium state, and thus a grain-growth microstructure [23]. 


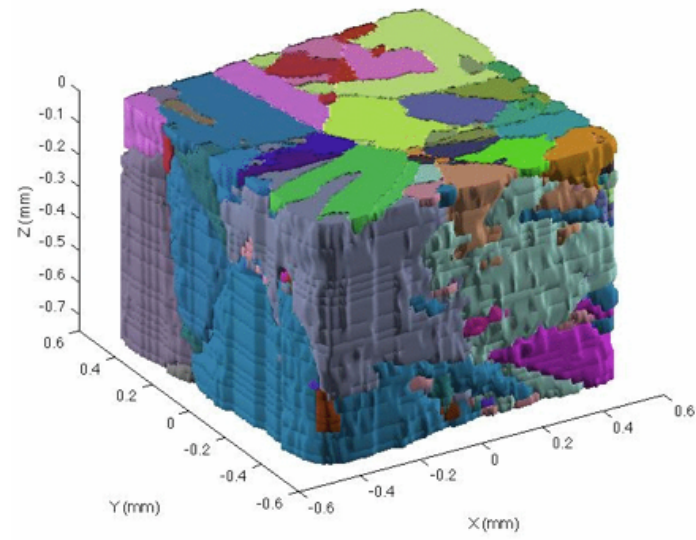

(a)

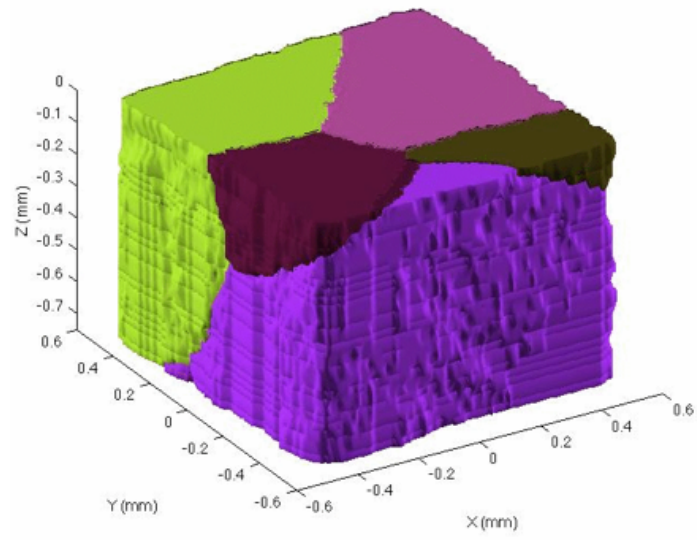

(b)

Figure 3: Near field high energy X-ray diffraction reconstructions. (a) Measured $\alpha$-phase orientation map, (b) calculated prior $\beta$-phase orientation map. (Reproduced from [21].)

\subsection{Simulation Methods}

The model employed in this study is an elastic-viscoplastic model embedded in a highly parallelized finite element framework. This method is capable of modeling large inelastic, quasi-static deformations of a polycrystal discretized into a finite element mesh. The deformation of the material is described using a set of constitutive equations, and we consider both an elastic response and inelastic response of a single crystal. Anisotropy is included in the elastic response, as well as the inelastic response, which considers only rate-dependent crystallographic slip restricted to dominant slip systems. Deformation twinning is not observed in Ti-6Al-4V due to its high aluminum content [1] and consequently was not modeled. Deformation is considered to be ductile and isothermal - no heat transfer or fracture models are included. Simulations are performed using the FEpX software package [24]. Here, a simplified summary of the model is described. A more complete description of the kinematics and configuration may be found in the FEpX theory and methods manual [24].

The equations for the elastic and plastic responses are written in a configuration reached by elastically unloading, without rotation, from the current, or spatial, configuration by the inverse of the elastic stretch, $\mathbf{v}^{e}$. The elastic portion of deformation is governed by Hooke's law,

$$
\boldsymbol{\tau}=\mathbf{C}(\mathbf{r}) \mathbf{e}^{e}
$$

where the Kirchhoff stress, $\boldsymbol{\tau}$, is related to the elastic strain, $\mathbf{e}^{e}$. The Kirchhoff stress is related to the Cauchy stress, $\boldsymbol{\sigma}$, by $\boldsymbol{\tau}=\operatorname{det}\left(\mathbf{v}^{e}\right) \boldsymbol{\sigma}$; the elastic strain is computed from the elastic stretch as $\mathbf{e}^{e}=\mathbf{v}^{e}-\mathbf{I}$, assuming small elastic strains [25,26]. The stiffness tensor, $\mathbf{C}$, is dependent on the symmetry of the crystal, and is reduced to reflect said symmetry [27]. Additionally, the stiffness tensor is a function of the orientation of the crystal, represented here using the Rodrigues' vector, $\mathbf{r}[28]$.

The plastic portion of deformation is governed by a rate-dependent restricted slip model. The plastic deformation rate, $\mathbf{D}^{P}$ (defined again in unloaded configuration), is a linear combination of the simple shearing modes defined by the symmetric portion of the Schmid tensors,

$$
\mathbf{D}^{P}=\sum_{k} \dot{\gamma}^{k} \mathbf{P}^{k}
$$




$$
\mathbf{P}^{k}=\operatorname{sym}\left(\mathbf{s}^{k} \otimes \mathbf{m}^{k}\right)
$$

where $\dot{\gamma}$ is the shearing rate. For each slip system, $k$, the Schmid tensor is defined as the dyadic product between a slip plane normal vector, $\mathbf{m}^{k}$, and a slip direction vector, $\mathbf{s}^{k}$. Additionally, the plastic deformation rate tensor may be reduced to a scalar value, or the effective plastic deformation rate:

$$
D_{e f f}^{P}=\sqrt{\frac{2}{3} \mathbf{D}^{P}: \mathbf{D}^{P}}
$$

Slip systems used in this study are shown in Table 2. The $\alpha$ phase utilizes three slip families in order to produce a sufficient number of independent slip systems to accommodate generalized plasticity.

\begin{tabular}{cccccc}
\hline Phase & Crystal Type & Slip System Name & Number of Systems & m & s \\
\hline$\alpha$ & HCP & Basal (b) & 3 & $\{0001\}$ & $\langle 11 \overline{2} 0\rangle$ \\
$\alpha$ & HCP & Prismatic (p) & 3 & $\{00 \overline{1} 0\}$ & $\langle 11 \overline{2} 0\rangle$ \\
$\alpha$ & HCP & Pyramidal $(\pi)$ & 12 & $\{00 \overline{1} 1\}$ & $\langle 11 \overline{2} 3\rangle$ \\
$\beta$ & BCC & - & 12 & $\{110\}$ & $\langle 111\rangle$ \\
\hline
\end{tabular}

Table 2: Slip systems used for $\alpha$ and $\beta$ crystallographic phases.

The kinetics of slip are defined using a power law relationship between the shearing rate of a given slip system and the resolved shear stress, $\tau^{k}$, on that system:

$$
\begin{aligned}
\dot{\gamma}^{k} & =\dot{\gamma}_{0}\left(\frac{\left|\tau^{k}\right|}{g^{k}}\right)^{\frac{1}{m}} \operatorname{sgn}\left(\tau^{k}\right) \\
\text { where } \tau^{k} & =\operatorname{tr}\left(\mathbf{P}^{k} \boldsymbol{\tau}^{\prime}\right)
\end{aligned}
$$

where $\boldsymbol{\tau}^{\prime}$ is the deviatoric portion of $\boldsymbol{\tau}$. The resolved shear stress on a slip system is scaled by the slip system strength, $g^{k}$. The fixed-state strain rate sensitivity is controlled by $m$, and $\dot{\gamma}_{0}^{k}$ is the fixed-state strain rate scaling coefficient. For a given element, $g^{k}$ is the same for all slip systems within a family.

Similarly, for a given element, each slip system within a family will evolve at the same rate. Evolution of a slip system's strength is modeled using a linear Voce type expression [29], while the saturation strength is a function of the sum of the slip system shear rates for a given element:

$$
\begin{gathered}
\dot{g}^{k}=\dot{\gamma} h_{0}\left(\frac{g_{s}^{k}(\dot{\gamma})-g^{k}}{g_{s}^{k}(\dot{\gamma})-g_{0}^{k}}\right) \\
\text { where } g_{s}^{k}(\dot{\gamma})=g_{s 0}^{k}\left(\frac{\dot{\gamma}}{\dot{\gamma}_{s 0}}\right)^{m^{\prime}} \text { and } \dot{\gamma}=\sum_{k}\left|\dot{\gamma}^{k}\right|
\end{gathered}
$$

The initial slip system strength and saturation strength are defined by $g_{0}^{k}$ and $g_{s}^{k}$, respectively, and $h_{0}$ is the strength hardening rate coefficient. The saturation strength is a function of the sum of the slip system shear rates for a given element, and is controlled by the initial saturation strength, $g_{s 0}^{k}$, the saturation strength strain rate scaling coefficient, $\dot{\gamma}_{s 0}$, and the saturation strength rate scaling exponent, $m^{\prime}$. For a given element, the saturation strengths of all slip systems within a family will evolve at the same rate. 
Model parameters were gathered from associated studies and literature. Single crystal elastic constants are outlined in Table 3. Single crystal elastic constants for the $\alpha$ phase were optimized in an associated study, utilizing a method based on minimizing the differences in discrete spherical harmonic modes of the lattice strain pole figures calculated from high energy X-ray diffraction experiments and from simulations [30]. Single crystal elastic constants for the $\beta$ phase were taken from literature $[4,31]$.

Values used for initial slip system strengths are outlined in Table 4. Relative initial slip system strengths for the $\alpha$ phase were also identified for our Ti-6Al-4V material using X-ray diffraction data in an associated study [32] and informed the parameters used in this study. The relative values of the strengths of the basal, prismatic and pyramidal slip systems are similar to values reported by others in the literature $[33,34]$ for Ti-6Al-4V. The basal and prismatic systems are comparable in strength while the pyramidal system is considerably stronger. The $\beta$ phase initial slip system strength was set equal to the initial slip system strengths of the basal and prismatic systems of the $\alpha$ phase, approximated to reflect current literature [35]. Other model parameters (Table 5) were chosen to limit the magnitude of hardening and effectively suppress evolution of the saturation strength. Both crystallographic phases are assumed to have the same parameters for the plastic flow kinetics and strain hardening, which were chosen to provide good agreement with the measured tensile behavior.

\begin{tabular}{ccccc}
\hline Phase & $C_{11}(\mathbf{G P a})$ & $C_{12}(\mathbf{G P a})$ & $C_{13}(\mathbf{G P a})$ & $C_{44}(\mathbf{G P a})$ \\
\hline$\alpha$ & 169.66 & 88.66 & 61.66 & 42.50 \\
$\beta$ & 133.10 & 95.10 & - & 42.70 \\
\hline
\end{tabular}

Table 3: Single crystal elastic constants for the $\alpha$ phase and $\beta$ phase.

\begin{tabular}{cccc}
\hline$g_{0, \beta}(\mathbf{M P a})$ & $g_{0, b}(\mathbf{M P a})$ & $g_{0, p}(\mathbf{M P a})$ & $g_{0, \pi}(\mathbf{M P a})$ \\
\hline 390 & 390 & 390 & 663 \\
\hline
\end{tabular}

Table 4: Initial slip system strengths for the $\alpha$ phase and $\beta$ phase.

\begin{tabular}{cccccc}
\hline$h_{0}(\mathbf{M P a})$ & $g_{s}(\mathbf{M P a})$ & $m$ & $m^{\prime}$ & $\dot{\gamma}_{0}\left(\mathbf{s}^{-1}\right)$ & $\dot{\gamma}_{s}\left(\mathrm{~s}^{-1}\right)$ \\
\hline 190 & 530 & 0.01 & 0.01 & 1.0 & $5 \times 10^{10}$ \\
\hline
\end{tabular}

Table 5: Plasticity parameters used for both the $\alpha$ phase and $\beta$ phase.

\section{Microstructure Instantiation}

\subsection{Geometric Instantiation}

The characterization of the $\beta$ annealed microstructure discussed in Section 2.1.2 provides a map of the prior $\beta$ phase grain structure from which the $\alpha$ phase colonies emerge upon cooling of the material. Instantiation of polycrystals for use in simulations will build on that knowledge, starting from prior $\beta$ phase grains, forming $\alpha$ phase colonies within grains, and finally $\beta$ phase lamella within $\alpha$ phase colonies. 


\subsubsection{Multilevel Tessellation Method}

Following this approach, a microstructure was generated by applying several levels of tessellations (i.e., a multilevel tessellation method). Tessellations were generated in a similar way at all levels by partitioning a domain of space, $D$, into cells. At the first level, $D$ corresponds to the body of the full polycrystal, while at subsequent levels, $D$ corresponds to each of the cells of the tessellations of the previous level. Tessellations described henceforth are either Laguerre tessellations or lamellar tessellations.

A Laguerre tessellation of a domain of space is constructed from a spatial distribution of seed points, $S_{i}(i \in[1, N])$, of non-negative weights, $w_{i}$. A cell, $C_{i}$, is associated with each seed point in the following manner:

$$
C_{i}=\left\{P(\mathbf{x}) \in D \mid\left\|P-S_{i}\right\|^{2}-w_{i}<\left\|P-S_{j}\right\|^{2}-w_{j} \forall j \neq i\right\}
$$

The resulting cells are convex polyhedra intersecting along planar faces, straight edges and vertices. The larger the weight of a seed (relative to the weights of other seeds), the bigger its associated cell. The actual size and shape of a cell, however, depend on the full set of seed positions and weights. A common way to define the positions and weights of the seeds is by sphere packing [36]. In this case, Laguerre tessellations are generated by evolving the seed positions and weights until desired (target) cell properties are obtained. In other words, Laguerre tessellations are obtained through the minimization of an objective function that quantifies the difference between the current cell properties and the target cell properties, and whose variables are the seed attributes.

It is possible to parametrize the cell size as the diameter of the sphere of equivalent volume, $d$, and the cell shape as the sphericity, $s$, which is equal to the ratio of the surface area of the sphere of equivalent volume to the surface area of the cell. The normalized diameter of a cell, $d_{n}$, is defined as $d /\langle d\rangle$, where ' \langle\rangle ' denotes the average over all cells. Let the target cumulative distribution functions of the normalized diameters and sphericities be written as $F_{d_{n}}$ and $F_{s}$, and their current counterparts be written as $F_{d_{n}}^{*}$ and $F_{s}^{*}$, respectively. The differences between the target and current distributions can be calculated in the same way for both variables and contribute equally to the objective function:

$$
\mathcal{O}=\int_{-\infty}^{+\infty} \frac{\left(F_{d_{n}}^{*}(x) \circ S-F_{d_{n}}(x) \circ S\right)^{2}}{F_{d_{n}}(x) \circ S\left(1-F_{d_{n}}(x) \circ S\right)} \mathrm{d} x+\int_{-\infty}^{+\infty} \frac{\left(F_{s}^{*}(x) \circ S-F_{s}(x) \circ S\right)^{2}}{F_{s}(x) \circ S\left(1-F_{s}(x) \circ S\right)} \mathrm{d} x
$$

where $\circ$ is the convolution product, and $S$ is a normal distribution of relatively small standard deviation (0.05) - introduced to facilitate optimization convergence. $\mathcal{O}$ takes a minimum value of zero when the current and target distributions are equal. This problem is solved using a gradientfree, non-linear optimization algorithm [37], by considering random seed positions and equal seed weights as as initial solution (which corresponds to a Voronoi tessellation).

A lamellar tessellation is generated by periodically slicing a domain with parallel planes. The width of the resulting lamellae is controlled by the plane positions and can be constant or not throughout the domain.

Both Laguerre tessellations and lamellar tessellations can be used to generate multilevel tessellations. At the first level, a tessellation is generated to produce primary cells. At the second level, each primary cell is divided into a new tessellation to produce secondary cells. This process can be repeated an arbitrary number of times until the desired microstructural representation is reached. The construction of a multilevel tessellation results in a collection of adjacent tessellations at the last level, which intersect along planar faces, straight edges and vertices of upper-level tessellations (this can be seen on Figure 5 which details a multilevel tessellation for the case of the $\beta$ annealed 
alloy). All the tessellation faces, edges and vertices that overlap at these intersections are eventually merged, and the resulting geometry is then subjected to regularization and meshing [20]. These methods are implemented in the Neper software package [38].

\subsubsection{Application to $\beta$ Annealed Microstructure}

Data collected from the near field diffraction experiments (Figure 3) were used as a starting point for informing the geometry of virtual microstructures. Distributions of grain size and sphericity are well established for grain-growth microstructures [23,39]. A Laguerre tessellation was used to represent the large, equiaxed prior $\beta$ phase grains. To facilitate the parametric study, the size distribution was altered from the grain-growth size distribution, such that it resulted in more uniform grain sizes. Figure 4 details the distributions describing the tessellation. The domain shape (rectangular prism) and aspect ratio (4:1) were chosen in order to mimic the gauge section of a physical specimen (Figure 5(a)).

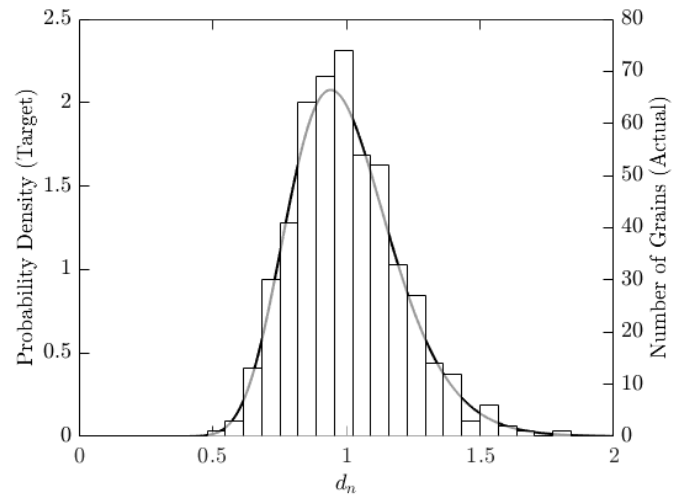

(a)

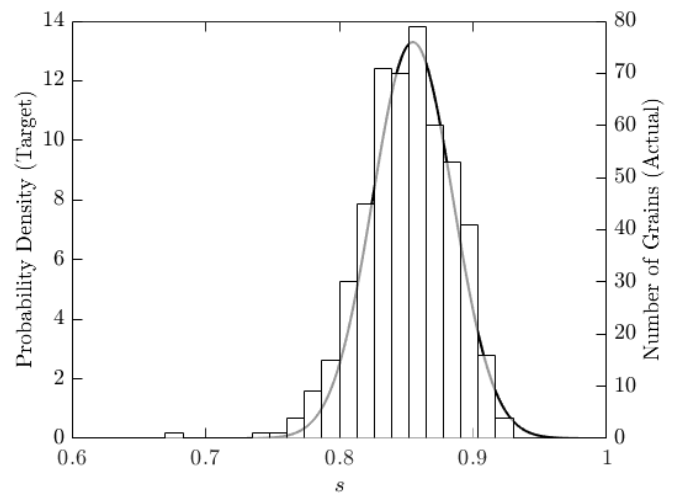

(b)

Figure 4: Target distributions (solid lines) and actual distributions (histograms) for (a) normalized equivalent diameter $\left(d_{n}\right)$ of the grains, and (b) sphericity $(s)$ of the grains. The distribution of the equivalent diameter of the grains is represented by a log-normal distribution with a mean of 1 and a standard deviation of 0.2 , while the distribution of sphericity of the grains is represented by a normal distribution with a mean of 0.855 and a standard deviation of 0.03 . An example tessellation with 500 grains was used for the actual distributions.

To represent the $\alpha$ phase colonies that transform within each prior $\beta$ phase grain, a second level tessellation was invoked. Using each prior $\beta$ phase grain as a domain, a Laguerre tessellation was performed where each new cell represents a colony (Figure 5(b)). Similar sphericity and size parameters as the first level tessellation were used for each second level tessellation. By using the prior $\beta$ phase grains as domains in which the $\alpha$ phase colonies form, $\alpha$ phase structures form the shape of a prior $\beta$ phase grain, and localized microtexturing is ensured when orientations are assigned.

Finally, remnant $\beta$ phase lamellae were represented using a third level of tessellation (Figure $5(\mathrm{c})$ ). As lamellar width was able to be altered, volume fractions of crystallographic phases were controlled by fixing the width of the $\beta$ lamellae and altering the width of the $\alpha$ phase in between lamellae. It has been observed that the flat portions of the lamellar planes are parallel to the (1100) plane of the $\alpha$ phase, or equivalently to the ( $\overline{1} 12)$ plane of the $\beta$ phase [1], in agreement with the Burgers orientation relationship between the $\alpha$ and $\beta$ phases (see Section 3.2). As such 
the lamellae were defined accordingly by relating the lamellar plane normals to either the grain or colony crystallographic orientation. Successive tessellation levels are concatenated to form the full geometric instantiation (Figure 5(d)).

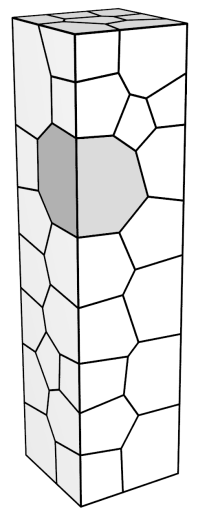

(a)

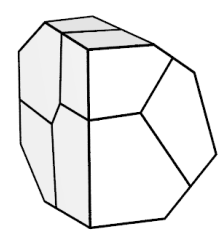

(b)

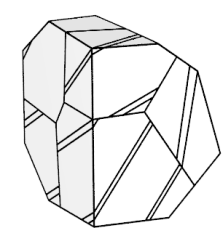

(c)

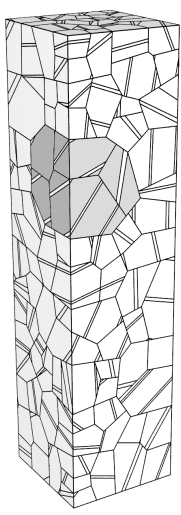

(d)

Figure 5: Example trilevel tessellation. (a) First level Laguerre tessellation in primary domain with example cell highlighted, (b) second level Laguerre tessellation using example first level Laguerre cell as domain (enlarged), (c) second level Laguerre cells discretized into lamellar planes (enlarged), (d) primary domain including all tessellation levels with example cell highlighted.

\subsection{Orientation Selection}

Orientations were selected with strict attention paid to localized microtexturing. While an experimental orientation distribution function could potentially be built from measured $\alpha$ phase orientations, such as those in Figure 3(a), random sampling from such a function would effectively eliminate localized microtexturing. As such, it is necessary to start from the pre-transformation structure and select orientations that would preserve this phenomenon. Ideally, an orientation distribution function for the reconstructed prior $\beta$ phase grains would be gathered experimentally. Presently, near field high energy X-ray diffraction experiments necessitate the use of small samples due to limitations in both time and current technological capabilities. As Figure 3(b) illustrates, the diffraction volume used captured only a small number of prior $\beta$ phase grains, far fewer than would be necessary to create a statistically significant orientation distribution function. Since the $\beta$ annealed microstructure was rolled and recrystallized above the transis temperature when it was composed entirely of cubic crystals, a Cube texture was assumed [40,41]. As such, an idealized orientation distribution function was generated using a spherical Gaussian-type distribution centered around the origin of Rodrigues' orientation space $[28,42]$, and is normalized over the cubic fundamental region, $\Omega$,

$$
\begin{aligned}
& A(\mathbf{r})=\frac{1}{\left(2 \pi \eta^{2}\right)^{\frac{3}{2}}} e^{-\frac{|\mathbf{r}|^{2}}{\eta^{2}}} \\
& \int_{\Omega} A(\mathbf{r}) \mathrm{d} v=1
\end{aligned}
$$

where $\eta$ controls the strength of texture. To provide a distribution with a reasonably strong texture, a value of $\eta=0.255$ was chosen, which creates an orientation distribution function with a maximal 
intensity of $\sim 13$ at the Cube orientation. Orientations for the prior $\beta$ phase grains are generated by randomly sampling from this idealized orientation distribution function, plotted in Figure 6(a).

The $\alpha$ phase colonies within prior $\beta$ phase grains have orientations that are strictly coupled to the orientation of the prior $\beta$ phase grains in which they form, obeying the Burgers orientation relationship:

$$
\begin{aligned}
& \{110\}_{\beta} \|\left(\begin{array}{llll}
0 & 0 & 0 & 1
\end{array}\right)_{\alpha} \\
& {[111]_{\beta} \|\left[\begin{array}{ll}
11 & \overline{2} 0]_{\alpha}
\end{array}\right.}
\end{aligned}
$$

For a given $\beta$ phase orientation, there are twelve possible $\alpha$ phase orientations that may arise as governed by Equation 10. To assign orientations for the $\alpha$ phase colonies that form within a prior $\beta$ grain, orientations were chosen at random from the twelve possibilities. In other words, it was assumed that no preference is given to variant selection. Figure 6(b) illustrates the idealized $\alpha$ phase texture that would arise from 10,000 prior $\beta$ phase grains transforming into 10,000 random $\alpha$ phase variants. Note the decrease in the strength of texture between the prior $\beta$ phase and the transformed $\alpha$ phase due to the single $\beta$ phase texture component being distributed among all the $\alpha$ variants. All calculations and plotting in Rodrigues' orientation space were performed using the ODFPF software package developed by the Deformation Processes Lab at Cornell University [43].

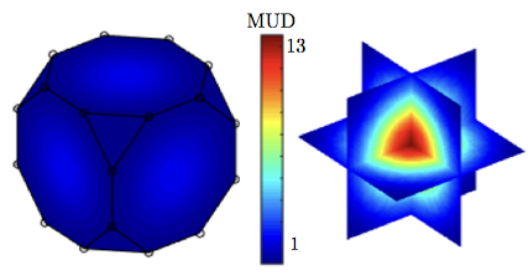

(a)

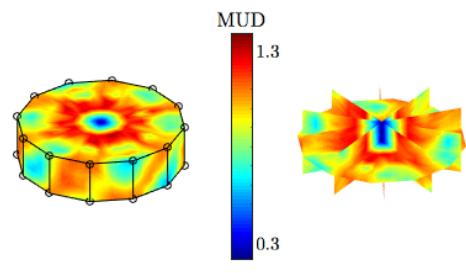

(b)

Figure 6: (a) Cube texture of prior $\beta$ phase grains plotted in the cubic symmetry fundamental region of Rodrigues' space, and (b) the resulting $\alpha$ phase texture assuming random variant selection plotted in the hexagonal symmetry fundamental region of Rodrigues' space. Scales presented as multiples of a uniform distribution (MUD).

\section{Simulations and Results}

\subsection{Simulation Suite}

A set of simulations was devised to study the effects the geometric features of the microstructure have on the mechanical behavior of the material. Both single phase ( $\alpha$ only) and dual phase simulations were conducted, and a full list is detailed in Table 6 . The main geometric features studied were the number of prior $\beta$ phase grains in a domain, the number of $\alpha$ phase colonies per grain, and the inclusion and width of remnant $\beta$ phase lamellae.

Single phase simulations were conducted to probe the effect of the number of grains in a domain, as well as the number of colonies per grain. Ignoring the remnant $\beta$ phase facilitated the study of the dominant $\alpha$ phase only. Five microstructure geometries with 40, 120, 200, 280, and 360 grains per domain and a single colony per grain were constructed. To provide details on variability for each of these five geometries, five simulations were conducted for each geometry, each time using a distinct orientation set. Similarly, five microstructure geometries with 40 grains and 5, 10, 
15, 20, and 25 colonies per grain were produced. Again, five simulations were conducted for each geometry using distinct orientation sets for each simulation. Example geometries for single phase instantiations can be seen in Figures 7 and 8.

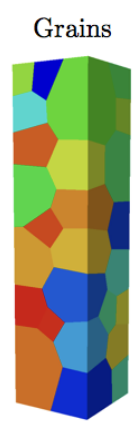

(a)

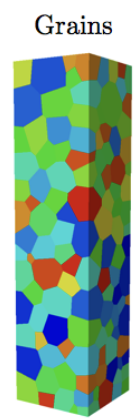

(b)

Figure 7: Examples of single phase geometries containing (a) 40 grains,and (b) 360 grains. Grains are colored arbitrarily.

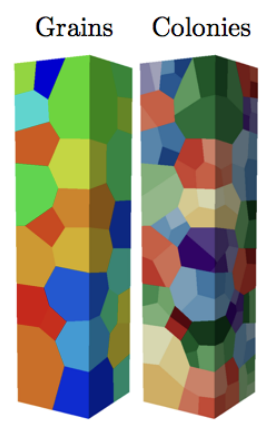

(a)

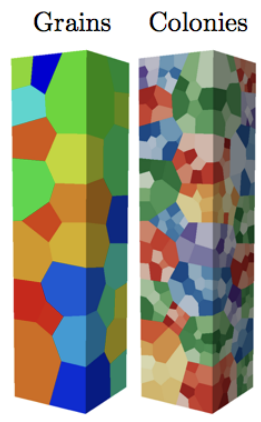

(b)

Figure 8: Examples of single phase geometries containing 40 grains with (a) 5 colonies per grain and (b) 25 colonies per grain. Grains are colored arbitrarily, while colonies are colored on monochromatic scales dependent on the grain in which they reside.

Investigating the inclusion of the $\beta$ phase in the simulations consisted mainly of studying the effects of the lamellae - in terms of their inclusion, size, and crystallographic orientation. While the simulation method described in Section 2.2 does not include an inherent length scale, the local stress field calculated using this method is influenced by the morphology of the microstructure and distribution of phases. As such, it is important to probe the effects of lamellar width on the local stress field, and in turn the macroscopic response. For each dual phase instantiation, volume fraction of the $\beta$ phase was fixed at $8 \% \pm 1 \%$. In investigating the effect of the size of the remnant $\beta$ phase lamellae, five base microstructure geometries were produced with 40 grains and a single colony per grain, and five base geometries were produced with 40 grains and five colonies per grain. Since the planar direction of the remnant $\beta$ phase lamella is dependent on the orientation set, each time a new orientation set was applied to a base geometry, the lamella would change. Each base geometry was used with five different orientation sets, and five lamellar widths $(0.10,0.08,0.06$, 0.04 , and 0.02 ) resulting in 50 geometric instantiations. In these simulations, a decrease in lamellar 
width leads to an increasing number of lamellae per grain in order to enforce phase volume fraction - which could also produce changes to the local stress field. Example geometries for dual phase instantiations containing lamella can be seen in Figure 9.

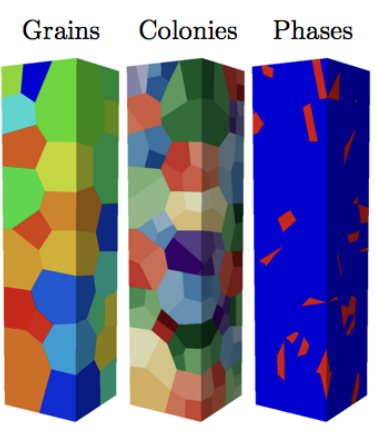

(a)

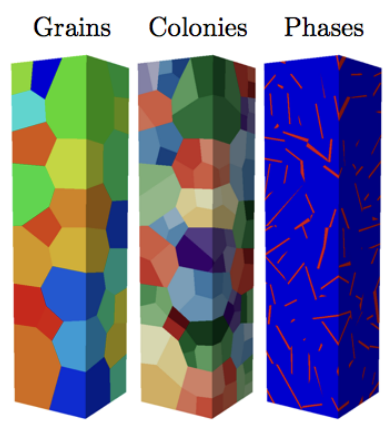

(b)

Figure 9: Examples of dual phase geometries containing 40 grains, 5 colonies per grain, and (a) lamella of width 0.10 and (b) lamella of width 0.02 . Grains are colored arbitrarily, while colonies are colored on monochromatic scales dependent on the grain in which they reside. Phase is colored using a binary color scale, where blue represents regions of $\alpha$ phase, and red represents regions of $\beta$ phase.

Five geometries were produced with 40 grains, 5, 10, 15, 20, and 25 colonies per grain, and a constant lamellar width of 0.10 . This set of geometries was simulated twice to gauge the effects of localized microtexturing on dual phase simulations. Each geometry was simulated once with orientations assigned such that the Burgers orientation relationship was properly enforced and once with the same orientation set assigned randomly to the geometry. Four Voronoi microstructures were produced with 500,1000, 1500, and 3000 grains, where random grains were assigned as $\beta$ phase to probe the effect of both the remnant $\beta$ phase's morphology (morphology consisted of no inter-grain colonies, nor association between plane normals and crystallographic direction), and thus localized microtexturing. Example geometries for dual phase Voronoi instantiations can be seen in Figure 10.

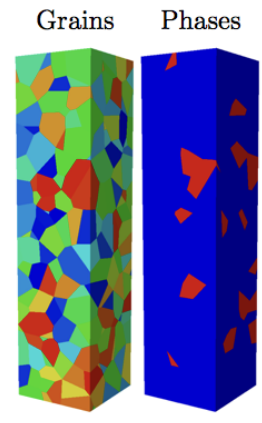

(a)

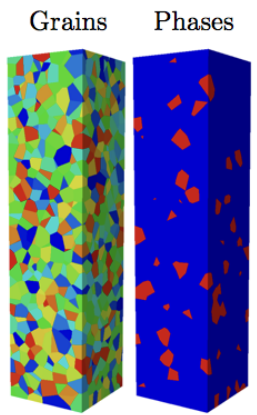

(b)

Figure 10: Examples of dual phase Voronoi geometries with (a) 500 total grains and (b) 3000 total grains. Phase is colored using a binary color scale, where blue represents regions of $\alpha$ phase, and red represents regions of $\beta$ phase. 


\begin{tabular}{ccccc}
\hline Grains & Colonies/Grain & Lamellar Width & Phases & Replications \\
\hline $40,120,200,280,360$ & 1 & - & $\alpha$ & 5 \\
40 & $5,10,15,20,25$ & - & $\alpha$ & 5 \\
40 & 1 & $0.10,0.08,0.06,0.04,0.02$ & $\alpha+\beta$ & 5 \\
40 & 5 & $0.10,0.08,0.06,0.04,0.02$ & $\alpha+\beta$ & 5 \\
40 & $5,10,15,20,25$ & 0.10 & $\alpha+\beta$ & 2 \\
$500,1000,1500,3000$ & 1 & - & $\alpha+\beta$ & 1 \\
\hline
\end{tabular}

Table 6: List of simulations conducted for the $\beta$ annealed microstructure. Replications refers to the number of orientation sets used for each geometry. For the dual phase $(\alpha+\beta)$ cases with a variable number of colonies per grain, instantiations were simulated twice - once enforcing the Burgers orientation relationship and once not.

To describe the number of grains in a cross section of a sample, a metric $\lambda$ is introduced, which is a ratio of the specimen's thickness to the average equivalent diameter (Section 3.1.1) of the specimen's grains [44]. Table 7 lists the calculated $\lambda$ values for the base geometries. As the total number of cells of distinct orientation are increased, the value of $\lambda$ increases (since the equivalent diameter of the grains must necessarily decrease). Previous studies suggest that an increase in the yield strength of a material is associated with an increase in $\lambda$ - specifically when $1<\lambda<3$ [44].

\begin{tabular}{ccc}
\hline Grains & Colonies/Grain & $\lambda$ \\
\hline $40,120,200,280,360$ & 1 & $1.81,2.67,3.13,3.62,3.96$ \\
40 & $5,10,15,20,25$ & $3.14,3.92,4.43,5.12,6.27$ \\
$500,1000,1500,3000$ & 1 & $3.99,5.18,6.33,8.86$ \\
\hline
\end{tabular}

Table 7: Average equivalent area ratio for different morphologies. Dual phase instantiations utilize the single phase geometries as base geometries, and as such have the same average equivalent area ratios.

\subsection{Instantiation and Data Reduction}

To showcase the steps from the formation of a virtual microstructure to the reduction of simulated data, a generic sample is shown. A geometric morphology is created for a sample (Section 3.1.2), during which orientations and phases are necessarily assigned - lamellae normals are needed for instantiation, and are known from the crystallographic orientations. Figure 11 shows an example orientation map and phase map for a dual phase complex morphology. 


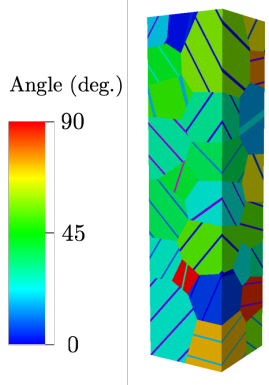

(a)

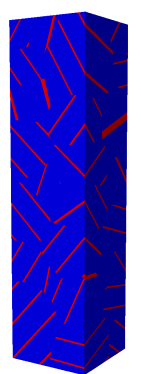

(b)

Figure 11: Example sample with 40 grains, 1 colony per grain, and a lamellar width of 0.02 , colored by (a) crystallographic orientation - specifically the deviation of the crystal $\left\{\begin{array}{lll}0 & 0 & 1\end{array}\right\}$ direction from the sample's loading direction, and (b) crystallographic phase (binary color scale where blue represents regions of $\alpha$ phase, and red represents regions of $\beta$ phase).

Data output from the deformation simulation is used to construct a sample stress strain curve - an example is shown in Figure 12. From this curve, the yield stress and ductility is calculated. Furthermore, data output from the simulations may be plotted against on the deformed geometry (Figure 13) to gain information about the progression of plasticity spatially. Information about the average mesh and simulation statistics can be viewed in Table 8. Below, aggregate and representative results deduced in this manner from each simulation are presented.

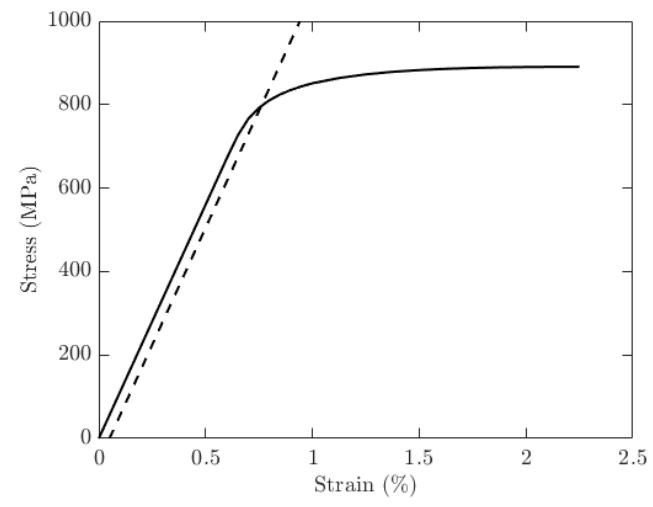

Figure 12: Example stress strain curve plotted to the ductility limit, with offset yield line (dashed). 


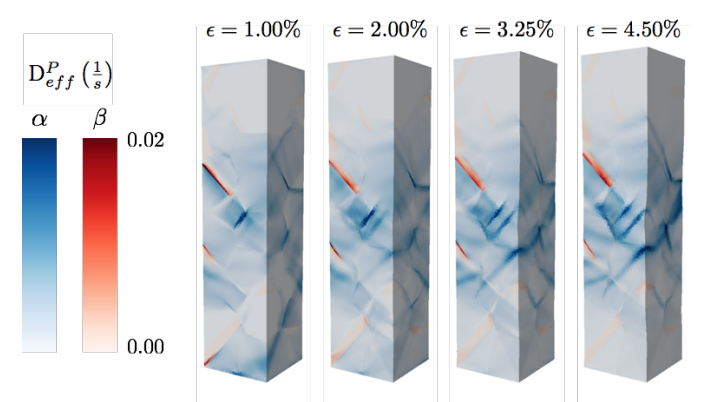

Figure 13: Example variable (effective plastic deformation rate) plotted spatially on a deformed mesh at various strains.

\begin{tabular}{cccccc}
\hline Grains & Colonies/Grain & Lamellar Width & NxPPN & Nodal Points & Duration \\
\hline $40,120,200,280,360$ & 1 & - & $8 \times 8$ & 160,000 & 4 \\
40 & $5,10,15,20,25$ & - & $8 \times 8$ & 140,000 & 4 \\
40 & 1 & $0.10,0.08,0.06,0.04$ & $16 \times 8$ & $1,250,000$ & 28 \\
40 & 1 & 0.02 & $32 \times 8$ & $2,800,000$ & $42^{*}$ \\
40 & 5 & $0.10,0.08,0.06,0.04$ & $16 \times 8$ & $1,260,000$ & 28 \\
40 & 5 & 0.02 & $32 \times 8$ & $3,220,000$ & $51^{*}$ \\
\hline
\end{tabular}

Table 8: Selected statistics for main simulation sets. Computer architecture is described by the number of computational nodes $(\mathrm{N})$ and the number of processors per node (PPN). Mesh size is described by the average number of nodal points, rounded to the nearest 10,000. Duration describes the average completion time, rounded to the nearest hour. Simulations marked with '*' used larger strain increments than other simulations, so as to reduce the total number of strain increments (by half) to reach the same final strain, effectively decreasing duration. Larger strain increments were not found to appreciably alter results.

\subsection{Simulation Result Trends}

For each simulation, the engineering strain was calculated from the initial sample length, total time at each load step, and velocity applied to the control surface. The engineering stress was calculated from the integrated load on the extended surface at each time step and the initial cross sectional area. A stress-strain history was calculated for each sample. Yield strength was calculated using a $0.1 \%$ offset method, and ductility was defined as the strain correlating to the maximum engineering stress - both matching the same methods used in the tensile experiments (Section 2.1.1).

\subsubsection{Yield Strength Trends}

Evolution of the yield strength as a function of geometric properties of the $\beta$ annealed microstructure are plotted here. Figure 14 shows yield strength trends for simulations conducted only with the $\alpha$ phase. Error bars represent variability in the data, calculated as the standard deviation for each data set. Figures 15 and 16 show the yield strength trends for the sets of dual phase simulations. Since multiple sets of simulations were not performed for the dual phase instantiations, no measure of variability is included. 


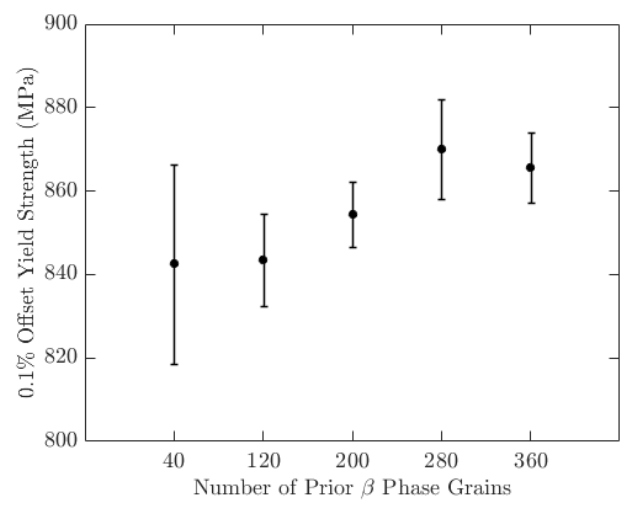

(a)

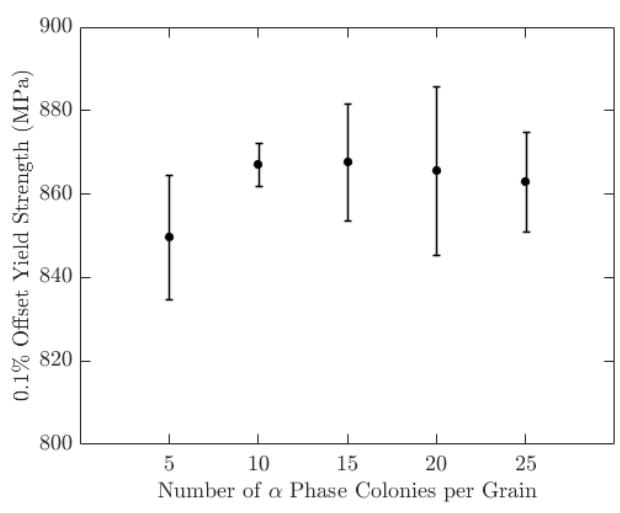

(b)

Figure 14: Yield strength trends of single phase simulations for changes in the (a) number of prior $\beta$ phase grains, and the (b) number of $\alpha$ phase colonies per grain (40 grains).

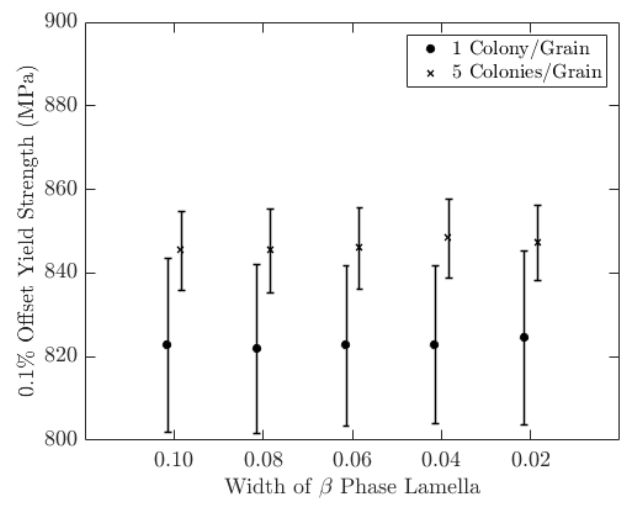

(a)

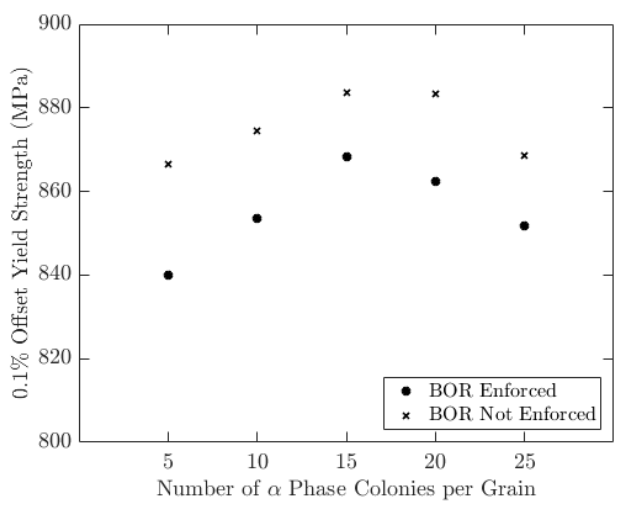

(b)

Figure 15: Yield strength trends for changes in the (a) width of remnant $\beta$ phase lamellae, and the (b) number of $\alpha$ phase colonies per grain with a constant lamellar thickness of $\mathrm{w}=0.10$. In (b), results are provided where the Burgers orientation relationship (BOR) was enforced or not. 


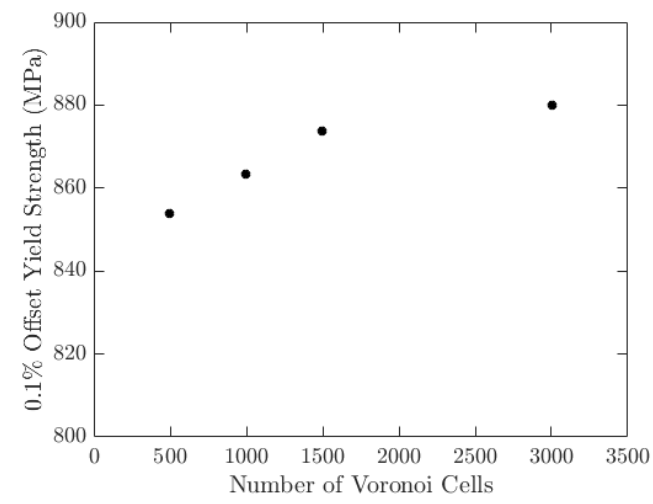

Figure 16: Yield strength trends for changes in the number of Voronoi cells in dual phase single level Voronoi tessellations. The number of Voronoi cells refers to the sum of both prior $\beta$ phase grains that have fully transformed to a single $\alpha$ variant, as well as grains composed solely of $\beta$ phase.

\subsubsection{Ductility and Hardening Trends}

Figures 17 through 19 detail ductility and hardening trends in a similar fashion to the yield strength trends presented in Section 4.3.1.

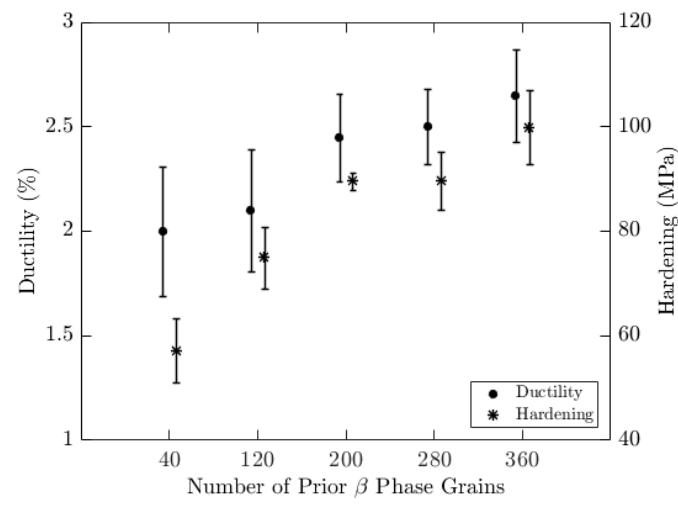

(a)

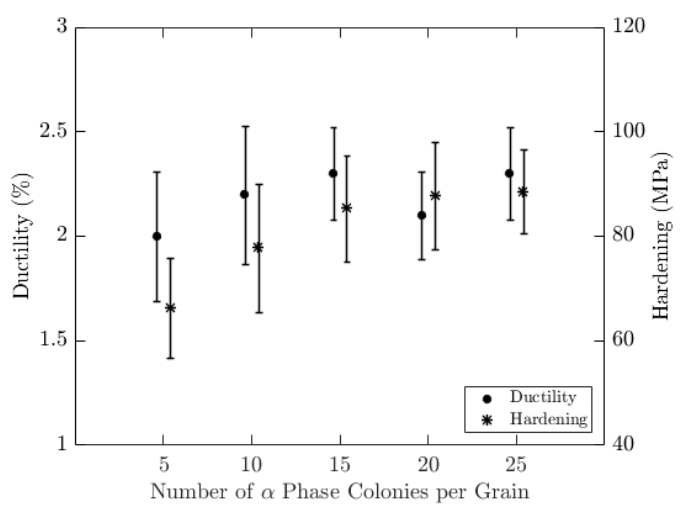

(b)

Figure 17: Ductility and hardening trends of single phase simulations for changes in the (a) number of prior $\beta$ phase grains, and the (b) number of $\alpha$ phase colonies per grain (40 grains). 


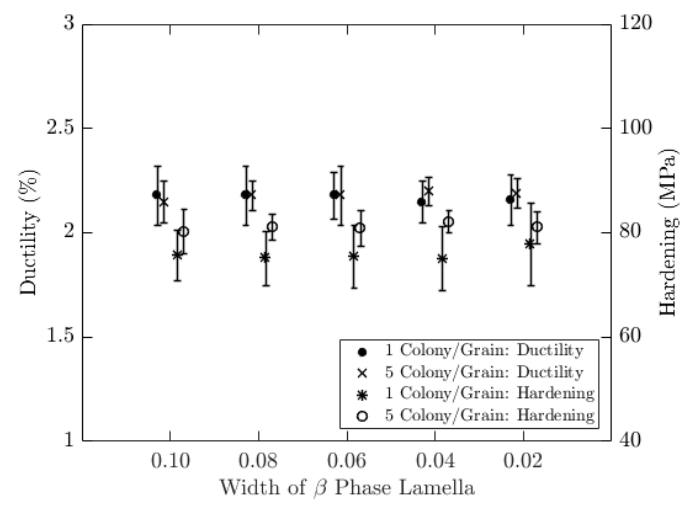

(a)

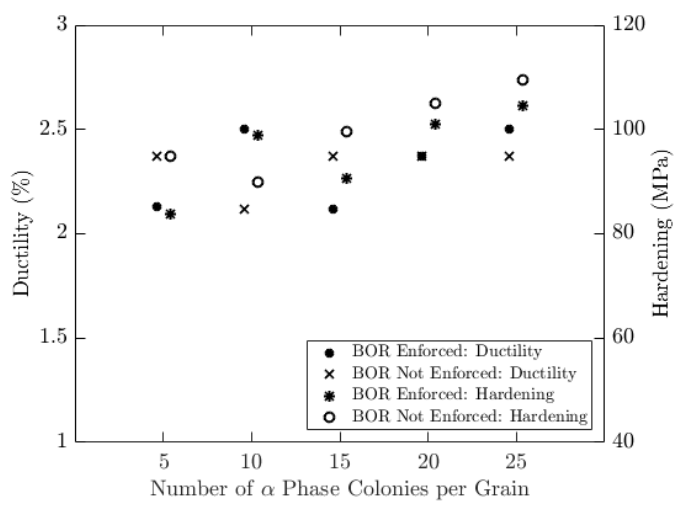

(b)

Figure 18: Ductility and hardening trends for changes in the (a) width of remnant $\beta$ phase lamellae, and the (b) number of $\alpha$ phase colonies per grain with a constant lamellar thickness of $\mathrm{w}=0.10$. In (b), results are provided where the Burgers orientation relationship (BOR) was enforced or not.

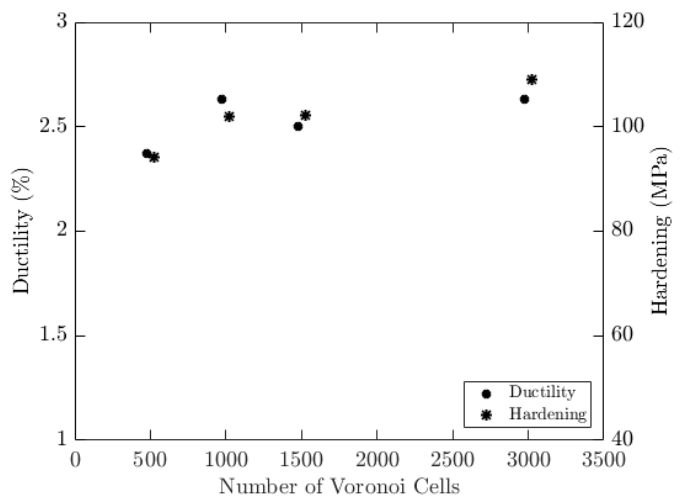

Figure 19: Ductility and hardening trends for changes in the number of grains in dual phase single level Voronoi tessellations. The number of Voronoi cells refers to the sum of both prior $\beta$ phase grains that have fully transformed to a single $\alpha$ variant, as well as grains composed solely of $\beta$ phase.

\section{Discussion}

\subsection{Yield Strength Trends}

For the single phase simulations, a strong positive correlation is seen between the number of prior $\beta$ phase grains and the offset yield strength (Figure 14(a)). Yield strength trends for increases in the number of grains correlate well with an increase in the specimen's $\lambda$ value found in Table 7 . Moreover, the yield strength is most variable when the number of grains is at its least. Looking at the yield strength as a function of the number of $\alpha$ phase colonies per grain reveals that the yield strength increases but saturates as the number of colonies per grain increases (though $\lambda$ increases as the number of colonies per grain increases). This is most likely attributed to the fact that there are only twelve $\alpha$ variants that can form for a given $\beta$ phase orientation, and as the number of colonies per grain is increased, the likelihood of new orientations being introduced in that domain 
decreases. Put simply, the yield strength saturates around ten $\alpha$ phase colonies per prior $\beta$ phase grain due to a saturation in localized microtexture.

For changes in the lamellar width, virtually no change in yield strength is observed (Figure 15(a)). Both simulation sets agree in this regard, though the simulations with 5 colonies per grain exhibit higher yield strengths than those with one, echoing the results of the single phase simulations. Inclusion of the remnant $\beta$ phase lowered the yield strength for both sets of simulations, though all values fell within the lower bound of the calculated variability for the single phase simulations. Figure 15(b) details yield strength trends for dual phase simulations where the number of colonies is variable - both with and without enforcement of localized microtexturing. For each simulation, when the Burgers orientation relationship was not enforced, the yield strength was higher, and by similar margins for each instantiation. Until 15 colonies per grain, an increase in the number of colonies per grain corresponds to an increase in the yield strength, after which a negative correlation is observed. Since only one simulation was run for each geometry, however, it is difficult to gauge overall trends.

For dual phase simulations conducted with Voronoi morphologies, the yield strength tends to be higher than dual phase simulations with a complex morphology (Figure 16). Additionally, it is evident that saturation occurs both at a higher level $(\sim 880 \mathrm{MPa})$ and with more cells of distinct orientation ( $\sim 1500$ cells) than the other dual phase simulations where the Burgers orientation relationship was enforced. These simulations consistently saturate at lower levels (maximum $\sim 860$ $\mathrm{MPa}$ ) and at a lower number of cells ( $\sim 400$ cells, ignoring $\beta$ lamellae). These values are both driven by the low number of grains used in the sample. However, the simulation set conducted with complex morphologies, but without the enforcement of localized microtexturing, was able to attain yield strengths comparable to those from the Voronoi morphologies (maximum $\geq 880 \mathrm{MPa}$ ).

\subsection{Ductility and Hardening Trends}

The calculated ductility exhibits many of the same trends as those for the calculated yield strengths. Single phase simulations exhibit the same positive trends - that is, as the number of grains is increased, the ductility increases (Figure 17(a)). Similarly, ductility increases as the number of colonies per grain is increased - though again, only marginally as the likelihood of distinct orientations decreases (Figure 17(b)). Qualitatively, these trends may be understood by inspecting the spatial distribution plastic deformation rate. Figures 20 and 21 detail representative results from the extreme cases of the single phase simulations by plotting the effective plastic deformation rate over the deformed virtual samples.

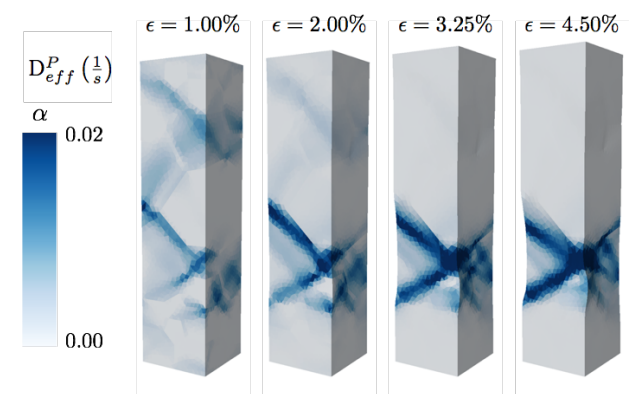

(a)

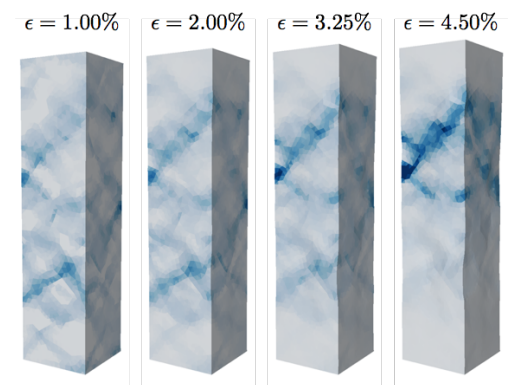

(b)

Figure 20: Representative results of the effective plastic deformation rate plotted at strains of $\epsilon=1.00 \%, \epsilon=2.00 \%, \epsilon=3.25 \%$, and $\epsilon=4.50 \%$ for (a) 40 grains and (b) 360 grains. 


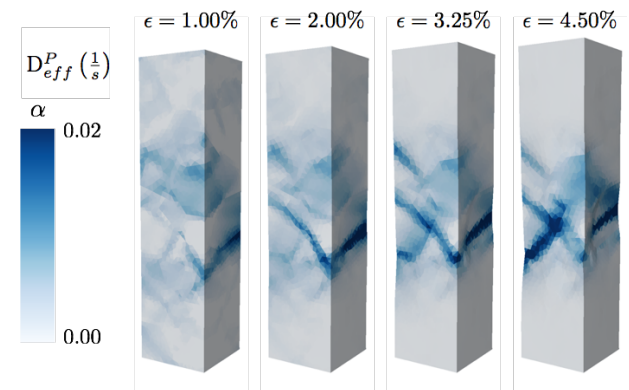

(a)

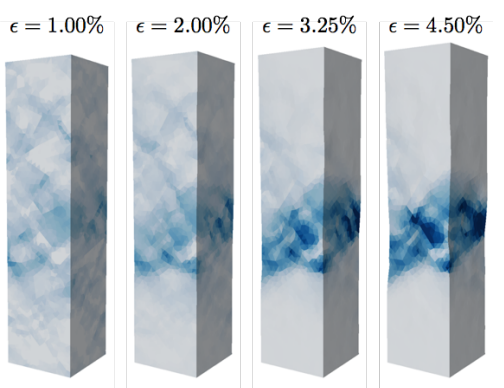

(b)

Figure 21: Representative results of the effective plastic deformation rate plotted at strains of $\epsilon=1.00 \%, \epsilon=2.00 \%, \epsilon=3.25 \%$, and $\epsilon=4.50 \%$ for 40 prior $\beta$ phase grains with (a) 5 colonies and (b) 25 colonies per grain.

As a region of plastically coalesces at a defined region spanning the cross section, the sample is no longer able to carry additional load. The formation of this yield band - or a region of localized plastic deformation - coincides with the ductility of a sample. Figure 20 showcases two deformation rate plots representing typical deformation characters for the limiting cases of grain variability. Deformation in the case with 40 grains localizes at a lower strain than in the case with 360 grains, reflecting the calculated ductility values. Similarly, Figure 21 shows similar plots for representative cases with 5 and 25 colonies per grain (40 grains). Deformation shows similar amounts of localization at the presented strains for both cases, again agreeing with the calculated ductility values. No strong trends in variability are apparent for either of the single phase simulation sets.

Adjusting the lamellar width for a sample has little to no effect on the ductility of the sample (Figure 18(a)). For both the cases with a single colony per grain, and the cases with 5 colonies per grain, the ductility remains effectively constant as lamellar width is changed. Figures 22 and 23 detail the spatial distribution of plastic deformation for the limiting cases of both simulation sets. For the cases with a single colony per grain, the distributions of plastic deformation rate are extremely similar for both simulations. For the cases with 5 colonies per grain, the distribution and intensities are nearly identical. Both of these qualitatively agree with the numerical values of the calculated ductilities. Changing the thickness of the lamellae does not alter the ductility of the sample, as evident by the weak effect it has on plasticity localization. 


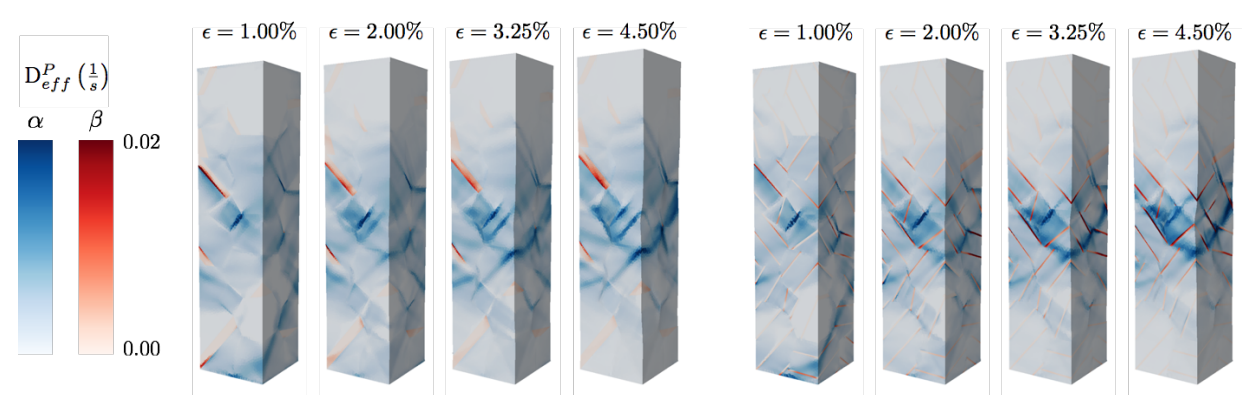

(a)

(b)

Figure 22: Representative results of the effective plastic deformation rate plotted at strains of $\epsilon=1.00 \%, \epsilon=2.00 \%, \epsilon=3.25 \%$, and $\epsilon=4.50 \%$ for 40 prior $\beta$ phase grains, $1 \alpha$ phase colony per grain, and lamellar widths of $(\mathrm{a}) \mathrm{w}=0.10$, and $(\mathrm{b}) \mathrm{w}=0.02$. Scales are identical for the two sets of plots.

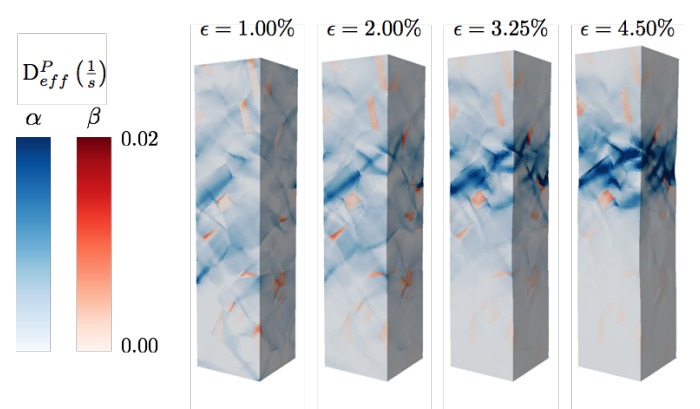

(a)

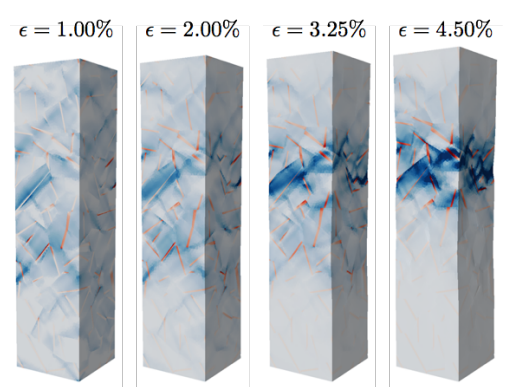

(b)

Figure 23: Representative results of the effective plastic deformation rate plotted at strains of $\epsilon=1.00 \%, \epsilon=2.00 \%, \epsilon=3.25 \%$, and $\epsilon=4.50 \%$ for 40 prior $\beta$ phase grains, $5 \alpha$ phase colonies per grain, and lamellar widths of $(\mathrm{a}) \mathrm{w}=0.10$, and $(\mathrm{b}) \mathrm{w}=0.02$. Scales are identical for the two sets of plots.

For the rest of the dual phase simulations, ductility is relatively constant, or no discernible trends are evident. For a complex morphology with a variable number of colonies per grain, a strong saturation is not immediately evident as was in the yield strength. Furthermore, loosening the restriction of the Burgers orientation relationship does not consistently increase or decrease the ductility. Dual phase simulations conducted with a Voronoi morphology, however, tend to have higher ductility values $(\sim 2.6 \%)$ than their counterparts conducted with complex morphologies and localized microtexturing (maximum $\sim 2.4 \%$ ) - similar to what is observed in the calculated yield strengths. This, too, may be attributed to the low number of grains present in these virtual samples, as evident by the values calculated from the single phase simulations.

Hardening trends tend to align well with ductility trends. Figures 17(a) and 17(b) detail hardening trends for single phase simulations. As the number of grains within a sample is increased, the amount of hardening also increases. Similar to yield and ductility trends, the amount of hardening saturates as the number of colonies per grain is increased past approximately 10 colonies per grain. Hardening in dual phase simulations also follows similar trends as ductility. Changes in lamellar width have little effect on hardening (Figure 18(a)), while enforcement of the Burgers orientation relationship tends to produce lower hardening than in simulations without enforcement 
(Figure 18(b)). Dual phase simulations conducted with a Voronoi morphology tend to exhibit more hardening than simulations conducted with complex morphologies.

\subsection{Effect of Morphology on Slip Activity}

The single mode of plastic deformation modeled is crystallographic slip, and thus it is the primary factor controlling the macroscopic yield and ductility of the sample. Inspecting slip activity at different points in the samples' deformation histories lends some insight into the bulk trends described above. Slip activity was probed in each phase independently, and calculated at various points of deformation. Activity for each phase is presented as a volume fraction of that phase with a certain number of slip systems active, calculated element by element. A slip system is considered active if its shear rate is above a threshold value, which here is related to the magnitude of the applied strain rate:

$$
\dot{\gamma} \begin{cases}<\frac{1}{10}\left|\dot{\epsilon}_{a p p}\right| & \text { inactive } \\ \geq \frac{1}{10}\left|\dot{\epsilon}_{a p p}\right| & \text { active }\end{cases}
$$

In all cases presented, results are averaged over the given simulation replications or simulation set so as to provide a representative result. Figure 24(a) details averaged results for all five simulations with 360 prior $\beta$ grains. Similarly, Figure 24(b) displays the average slip activity at various strains for the $25 \alpha$ colony simulations. Figures 25 through 29 detail slip results for all dual phase simulation sets. Averages were calculated using all instantiations per set.

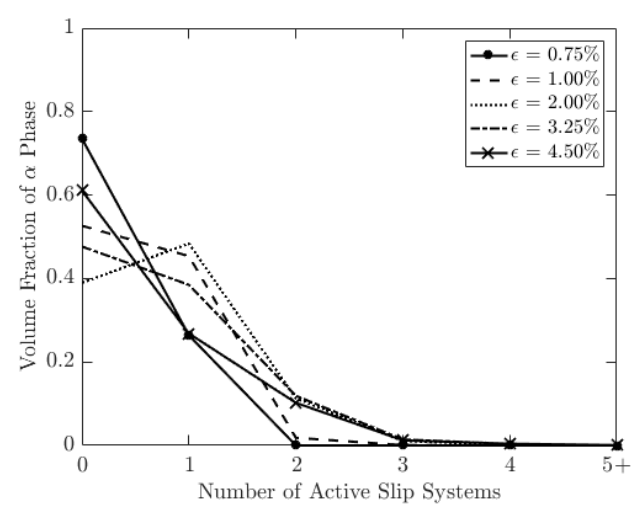

(a)

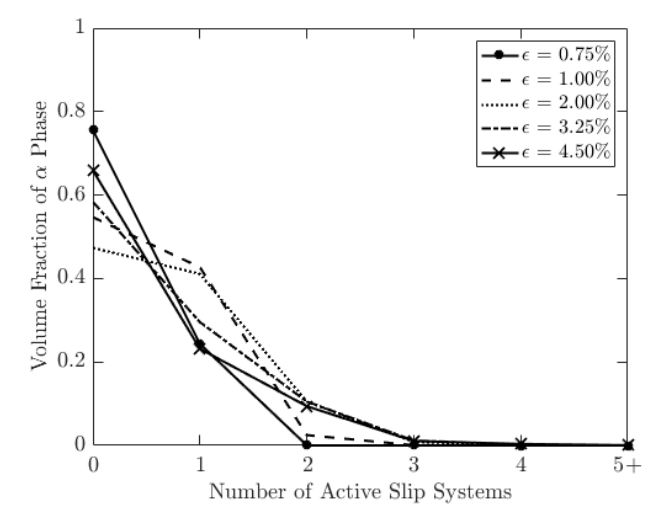

(b)

Figure 24: Average slip system activity for single phase simulations with (a) 360 prior $\beta$ phase grains with a single colony per grain, and (b) 40 prior $\beta$ phase grains with $25 \alpha$ phase colonies per grain. 


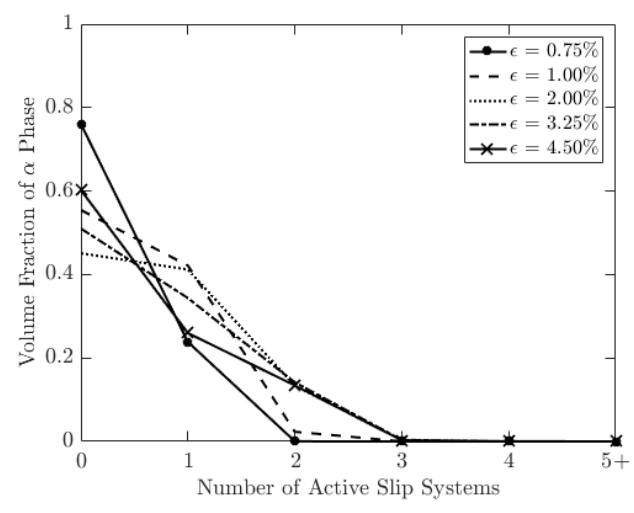

(a)

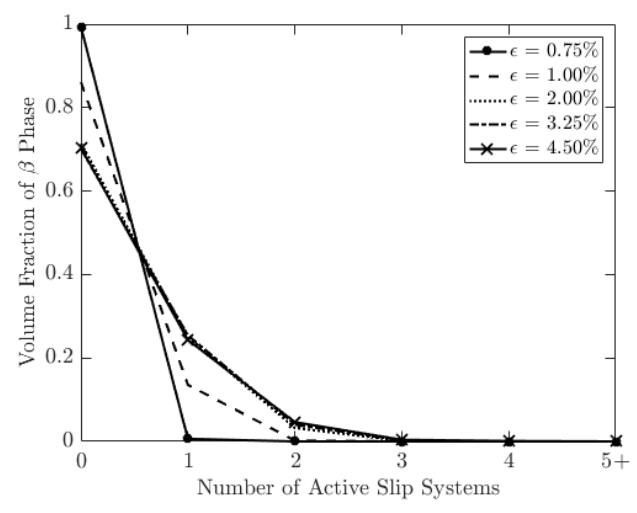

(b)

Figure 25: Average slip system activity in the (a) $\alpha$ phase and (b) $\beta$ phase for simulations with 40 prior $\beta$ phase grains, $1 \alpha$ phase colony per grain, and variable lamellar thickness.

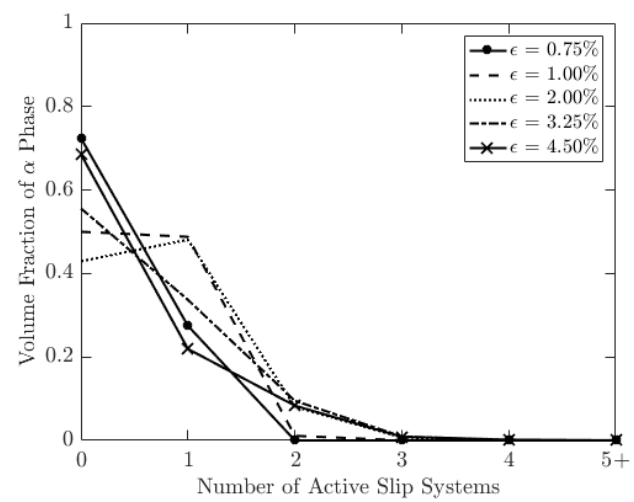

(a)

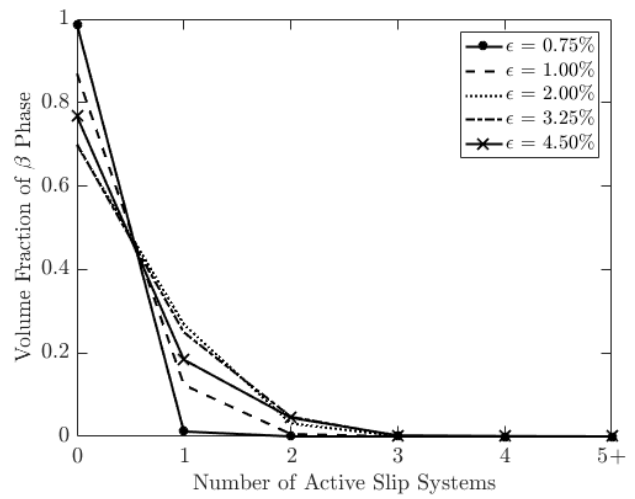

(b)

Figure 26: Average slip system activity in the (a) $\alpha$ phase and (b) $\beta$ phase for simulations with 40 prior $\beta$ phase grains, $5 \alpha$ phase colonies per grain, and variable lamellar thickness. 


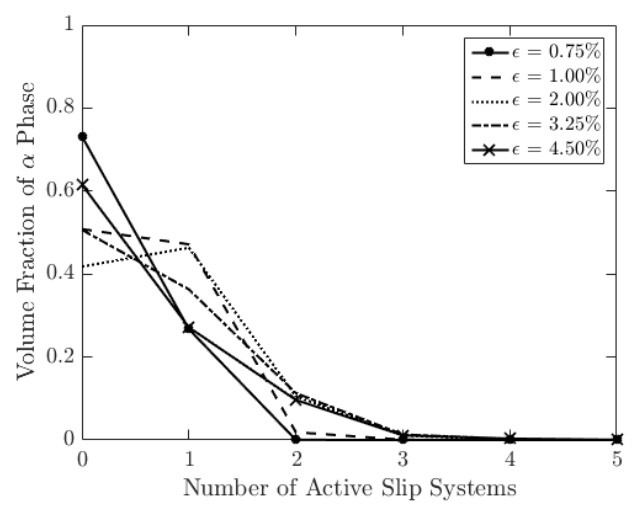

(a)

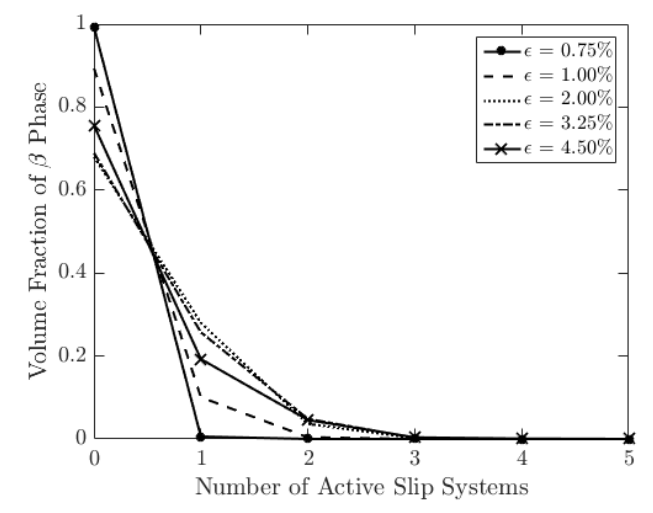

(b)

Figure 27: Average slip system activity in the (a) $\alpha$ phase and (b) $\beta$ phase for simulations with 40 prior $\beta$ phase grains, variable $\alpha$ phase colonies per grain, and lamellae thickness of $\mathrm{w}=0.10$.

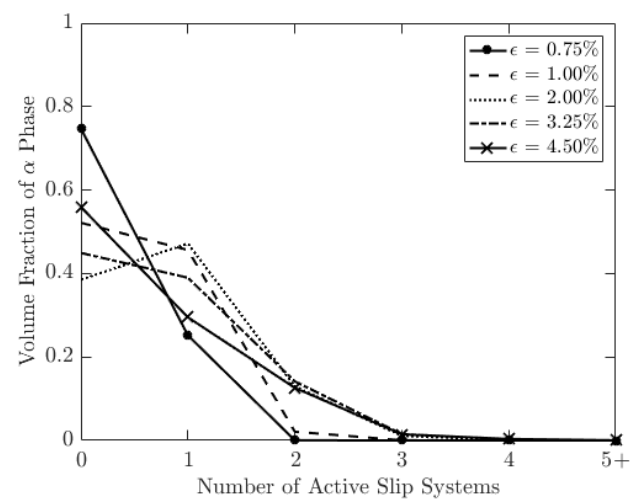

(a)

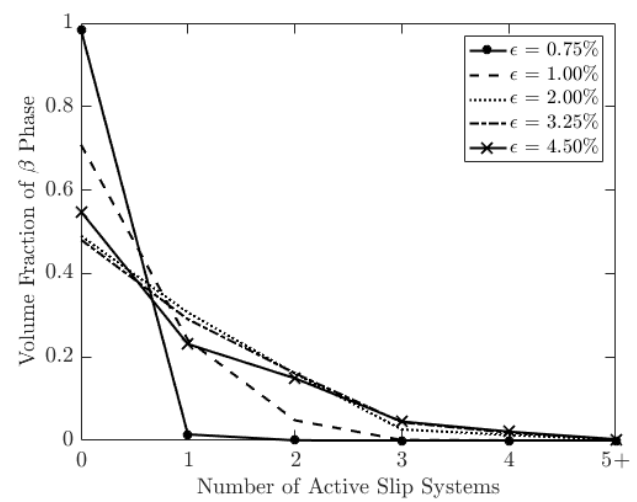

(b)

Figure 28: Average slip system activity in the (a) $\alpha$ phase and (b) $\beta$ phase for dual phase Voronoi tessellations. 


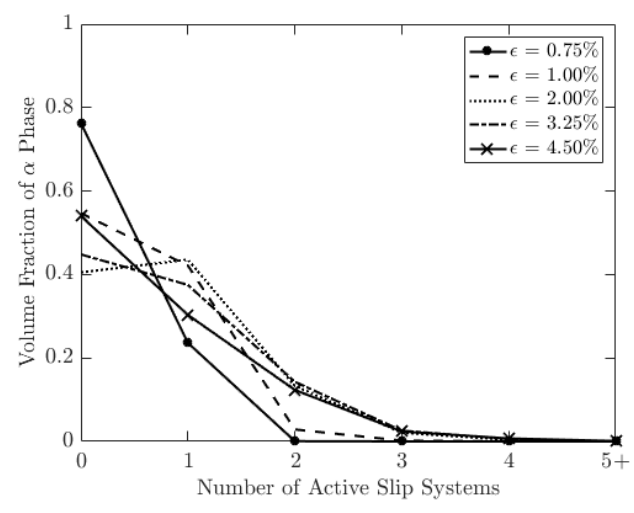

(a)

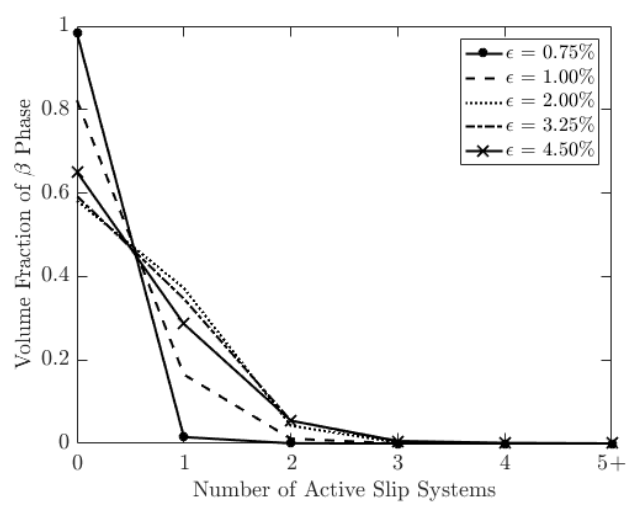

(b)

Figure 29: Average slip system activity in the (a) $\alpha$ phase and (b) $\beta$ phase for simulations with 40 prior $\beta$ phase grains, variable $\alpha$ phase colonies per grain, and a lamellae thickness of $\mathrm{w}=0.10$, without the enforcement of the Burgers orientation relationship (no localized microtexturing).

When comparing only the slip activity in the $\alpha$ phase for both single phase and dual phase simulations, the character of slip is relatively consistent, and some trends are evident. Firstly, polyslip (3 or more slip systems active) was effectively nonexistent, with regions exhibiting it representing at most less than $5 \%$ of the sample's $\alpha$ phase volume at any point in deformation. At most, about $60 \%$ of the $\alpha$ phase deformed plastically, before deactivating upon plasticity localization. Early in deformation, the overwhelming majority of the active volume deformed through single slip. Late in deformation, the volume of the regions that deformed by dual slip increased, though even then only to about half of the volume of that exhibiting single slip.

For dual phase simulations with complex morphologies and localized microtexturing, slip in the $\beta$ phase activated later in deformation than the $\alpha$ phase, and overall activity was lower than that of the $\alpha$ phase (Figures 25 through 27). While the $\alpha$ phase activated before macroscopic yield $(\epsilon=0.5 \%)$, the $\beta$ phase was found to be entirely elastic at that same point. At peak, only $30 \%$ of the $\beta$ phase was inelastic - half that of the $\alpha$ phase. Further, those $\beta$ phase regions that were plastically deforming exhibited almost entirely activity of only a single slip system. Again, polyslip was effectively nonexistent.

Dual phase simulations conducted with a Voronoi morphology, however, exhibited $\beta$ phase slip activity markedly different than that of the previous simulations. While the $\beta$ phase still activated later than the $\alpha$ phase, as it did in the instantiations with complex morphologies, much more of the $\beta$ phase volume fraction became active - nearly to the same level as the $\alpha$ phase. At peak, more than $50 \%$ of the $\beta$ phase became plastically active, nearly double what is seen in simulations constructed with complex morphologies. Furthermore, while single slip was the still dominant mode of plastic deformation in the $\beta$ phase, double slip occurred in a significant volume at each point in deformation, even nearly equalling the volume deforming by single slip later in deformation. Though small, polyslip was non-negligible in the $\beta$ phase $(\sim 15 \%$ at its peak), unlike any other simulated slip character for either phase.

Simulations conducted with a complex morphology without localized microtexturing exhibited a slight increase in $\beta$ phase slip activity (Figure 29). At peak, more than $40 \%$ of the $\beta$ phase had activated - a $10 \%$ increase from the same morphologies with localized microtexturing. This increase in plasticity, however, was nearly entirely single slip - no large increase in double slip or polyslip was witness when localized microtexturing was relaxed. This implies that localized microtexturing 
and morphological constraints both contribute to restricted slip in the $\beta$ phase when compared to simulations with a generic morphology.

\section{Conclusion}

A parameter study of the geometric features of $\beta$ annealed Ti-6Al-4V is presented. Complex representations are instantiated by means of a multilevel tessellation method and include details of the high temperature morphology (prior $\beta$ phase grains). This method allows for individual parameters controlling the geometry to be altered independently and also allows localized microtexturing to be enforced. The intent is to understand better the role of mechanical constraints introduced by the microstructural geometry on an aggregate's strength and ductility. The simulations demonstrated that:

- The calculated yield strength for a simulation is governed by the number of prior $\beta$ phase grains in an instantiation, as well as the number of $\alpha$ phase colonies per grain - but with the latter only until the probability of new variants decreased and localized microtexturing became saturated. Inclusion of remnant $\beta$ phase lamellae tended to decrease the yield strength, but lamellar width had little to no effect.

- The calculated ductility and hardening for a simulation are governed by the same geometric features as the yield strength. The point of ductility was seen to correspond with the formation of a localized band of plastic deformation, which generally formed more readily when there were less cells of distinct orientation through a cross section. Again, lamellar width had little to no effect on the macroscopic ductility or hardening.

- Slip activity in the $\beta$ phase was restricted in simulations conducted with complex morphologies and localized microtexturing (enforcement of the Burgers orientation relationship), and both morphology and texture are shown to be influential in this phenomenon. Dual phase simulations conducted with generic Voronoi tessellations exhibited higher strength and ductility, and more hardening, than those conducted with complex morphologies, as well as a high amount of $\beta$ phase slip activity.

\section{Acknowledgements}

Funding for this study was provided by the Office of Naval Research under grant N00014-12-10399, as well as the Cornell High Energy Synchrotron Source (CHESS) under NSF award DMR1332208. Professor Jim Williams is thanked for his expertise and acquisition of necessary materials from TIMET. Dr. Euan Wielewski is thanked for his advice, conducting the tensile experiments in this paper, and sharing data of the near field high energy X-ray diffraction experiment. Donald Boyce is thanked for his work in estimating constitutive model parameters.

\section{References}

[1] G. Lütjering and J.C. Williams. Titanium. Engineering Materials and Processes. Springer, Berlin; New York, 2nd edition, 2007.

[2] R. Ding, Z.X. Guo, and A. Wilson. Microstructural evolution of a Ti-6Al-4V alloy during thermomechanical processing. Materials Science and Engineering: A, 327(2):233-245, 2002. 
[3] S. L. Semiatin, S. L. Knisley, P. N. Fagin, D. R. Barker, and F. Zhang. Microstructure evolution during alpha-beta heat treatment of Ti-6Al-4V. Metallurgical and Materials Transactions A, 34(10):2377-2386, 2003.

[4] A Kelly and G.W. Groves. Crystallography and Crystal Defects. Prentice Hall, Reading, 1970.

[5] C. Tomé and U.F. Kocks. The yield surface of h.c.p. crystals. Acta Metallurgica, 33(4):603-621, 1985 .

[6] R.J. Asaro and A. Needleman. Texture development and strain hardening in rate dependent polycrystals. Acta Metallurgica, 33(6):923-953, 1985.

[7] E.B. Marin and P.R. Dawson. On modelling the elasto-viscoplastic response of metals using polycrystal plasticity. Computer Methods in Applied Mechanics and Engineering, 165(1-4):1$21,1998$.

[8] E.B. Marin and P.R. Dawson. Elastoplastic finite element analyses of metal deformations using polycrystal constitutive models. Computer Methods in Applied Mechanics and Engineering, 165(1-4):23-41, 1998.

[9] G.B. Sarma and P.R. Dawson. Effects of interactions among crystals on the inhomogeneous deformations of polycrystals. Acta Materialia, 44(5):1937-1953, 1996.

[10] D. Raabe, Z. Zhao, and W. Mao. On the dependence of in-grain subdivision and deformation texture of aluminum on grain interaction. Acta Materialia, 50(17):4379-4394, 2002.

[11] M. Zhang, J. Zhang, and D.L. McDowell. Microstructure-based crystal plasticity modeling of cyclic deformation of Ti-6Al-4V. International Journal of Plasticity, 23(8):1328-1348, 2007.

[12] M.J. Philippe, M. Serghat, P. Van Houtte, and C. Esling. Modelling of texture evolution for materials of hexagonal symmetry - II. application to zirconium and titanium $\alpha$ or near $\alpha$ alloys. Acta Metallurgica et Materialia, 43(4):1619-1630, 1995.

[13] N.R. Barton and P.R. Dawson. Lattice misorientations in titanium alloys: modeling the origins of defects. International Journal of Forming Processes, 5(2-3-4):189-201, 2002.

[14] M. Obstalecki, S.L Wong, P.R. Dawson, and M.P. Miller. Quantitative analysis of crystal scale deformation heterogeneity during cyclic plasticity using high-energy X-ray diffraction and finite-element simulation. Acta Materialia, 75:259-272, 2014.

[15] S.L. Wong, M. Obstalecki, M.P. Miller, and P.R. Dawson. Stress and deformation heterogeneity in individual grains within polycrystals subjected to fully reversed cyclic loading. Journal of the Mechanics and Physics of Solids, 79:157-185, 2015.

[16] M.P. Echlin, A. Mottura, C.J. Torbet, and T.M. Pollock. A new TriBeam system for threedimensional multimodal materials analysis. Review of Scientific Instruments, 83(2):023701, 2012 .

[17] U. Lienert, S. F. Li, C. M. Hefferan, J. Lind, R. M. Suter, J. V. Bernier, N. R. Barton, M. C. Brandes, M. J. Mills, M. P. Miller, B. Jakobsen, and W. Pantleon. High-energy diffraction microscopy at the advanced photon source. JOM, 63(7):70-77, 2011. 
[18] O. Watanabe, H.M. Zbib, and E. Takenouchi. Crystal plasticity: micro-shear banding in polycrystals using voronoi tessellation. International Journal of Plasticity, 14(8):771-788, 1998.

[19] F. Barbe, L. Decker, D. Jeulin, and G. Cailletaud. Intergranular and intragranular behavior of polycrystalline aggregates. Part 1: F.E. model. International Journal of Plasticity, 17(4):513$536,2001$.

[20] R. Quey, P.R. Dawson, and F. Barbe. Large-scale 3D random polycrystals for the finite element method: Generation, meshing and remeshing. Computer Methods in Applied Mechanics and Engineering, 200(17-20):1729-1745, 2011.

[21] E. Wielewski, D.B. Menasche, P.G. Callahan, and R.M. Suter. Three-dimensional $\alpha$ colony characterization and prior- $\beta$ grain reconstruction of a lamellar Ti-6Al-4V specimen using nearfield high-energy X-ray diffraction microscopy. Journal of Applied Crystallography, 48(4):11651171, 2015.

[22] M.G. Glavicic, P.A. Kobryn, T.R. Bieler, and S.L. Semiatin. A method to determine the orientation of the high-temperature beta phase from measured EBSD data for the low-temperature alpha phase in Ti-6Al-4V. Materials Science and Engineering: A, 346(1-2):50-59, 2003.

[23] D.J. Rowenhorst, A.C. Lewis, and G. Spanos. Three-dimensional analysis of grain topology and interface curvature in a $\beta$-titanium alloy. Acta Materialia, 58:5511-5519, 2010.

[24] P.R. Dawson and D.E. Boyce. FEpX - Finite Element Polycrystals: Theory, Finite Element Formulation, Numerical Implementation and Illustrative Examples, 2015.

[25] A.F. Bower. Applied Mechanics of Solids. CRC Press, 2010.

[26] R.W. Ogden. Non-linear Elastic Deformations. Dover Publications, 1997.

[27] J.F. Nye. Physical Properties of Crystals: Their Representation by Tensors and Matrices. Clarendon Press ; Oxford University Press, 1984.

[28] F.C. Frank. Orientation Mapping. MRS Bulletin, 13(03):24-31, 1988.

[29] U. F. Kocks, C. N. Tomé, and H.R. Wenk. Texture and anisotropy: preferred orientations in polycrystals and their effect on materials properties. Cambridge Univ. Press, Cambridge, 1st edition, 2000.

[30] E. Wielewski, D.E. Boyce, J.S. Park, M.P. Miller, and P.R. Dawson. A methodology to determine the elastic moduli of crystals by matching experimental and simulated lattice strain pole figures using discrete harmonics. Acta Materialia, in press.

[31] N.R. Barton and P.R. Dawson. On the spatial arrangement of lattice orientations in hotrolled multiphase titanium. Modelling and Simulation in Materials Science and Engineering, 9(5):433-463, 2001.

[32] P.R. Dawson, D.E. Boyce, J.S. Park, E. Wielewski, and M.P. Miller. Determining the slip system strengths in hcp titanium alloys using discrete harmonic analyses of lattice strain pole distributions. In preparation.

[33] I.P. Jones and W.B. Hutchinson. Stress-state dependence of slip in Titanium-6Al-4V and other H.C.P. metals. Acta Materialia, 23(9):951-968, 1981. 
[34] F. Bridier, D.L. McDowell, P. Villechaise, and J. Mendez. Crystal plasticity modeling of slip activity in Ti-6Al-4V under high cycle fatigue loading. International Journal of Plasticity, 25(6):1457-1485, 2009.

[35] J.R. Mayeur and D.L. McDowell. A three-dimensional crystal plasticity model for duplex Ti-6Al-4V. International Journal of Plasticity, 23(9):1066-1082, 2007.

[36] Z. Fan, Y. Wu, X. Zhao, and Y. Lu. Simulation of polycrystalline structure with voronoi diagram in laguerre geometry based on random closed packing of spheres. Computational Materials Science, 29:301-308, 2004.

[37] S.G. Johnson. The NLopt nonlinear-optimization package. http://ab-initio.mit.edu/ nlopt.

[38] R. Quey. Neper: polycrystal generation and meshing (version 3.0). http://neper. sourceforge.net, 2016.

[39] A. Spettl, T. Werz, C.E. Krill, and V. Schmidt. Parametric representation of 3d grain ensembles in polycrystalline microstructures. Journal of Statistical Physics, 154:913-928, 2014.

[40] J.H. Keeler and A.H. Geisler. Preferred Orientations in Rolled and Annealed Titanium. Journal of Metals, 8:80-90, 1956.

[41] P.D. Wu, S.R. MacEwen, D.J. Lloyd, and K.W. Neale. Effect of cube texture on sheet metal formability. Materials Science and Engineering: A, 364(1-2):182-187, 2004.

[42] A. Kumar and P.R. Dawson. Modeling crystallographic texture evolution with finite elements over neo-Eulerian orientation spaces. Computer Methods in Applied Mechanics and Engineering, 153(3-4):259-302, 1998.

[43] P.R. Dawson and D.E. Boyce. OdfPf Library by The Deformation Processes Lab at Cornell University. https://anisotropy.mae.cornell.edu/onr/Matlab/matlab.html, 2004.

[44] P.J.M. Janssen, Th.H. de Keijser, and M.G.D. Geers. An experimental assessment of grain size effects in the uniaxial straining of thin $\mathrm{Al}$ sheet with a few grains across the thickness. Materials Science and Engineering: A, 419(1-2):238-248, March 2006. 